Harmonics Suppression in Electromagnets with Application to the Als Storage Ring Corrector Magnet Design*

\author{
R. D. Schlueter
}

Advanced Light Source

Accelerator and Fusion Research Division

Lawrence Berkeley Laboratory

University of California

Berkeley, CA 94720

January 28, 1991
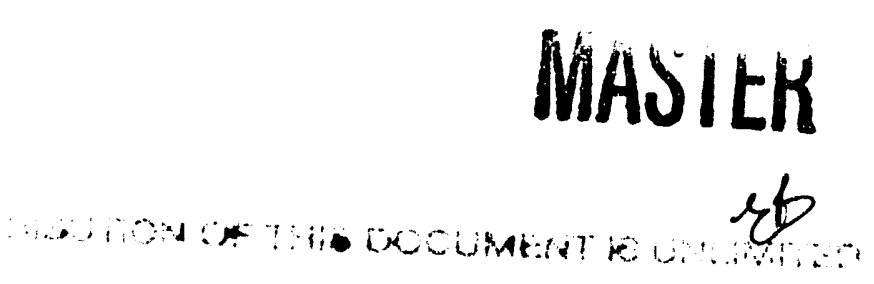

This work was supported by the Director, Office of Energy Reseanch, Office of Basic Energy Sciences, Materials Sciences Division of the U.S. Departenent of Energy, under Contract No. DE-AC03-76SF00098 


\title{
Harmonics Suppression in Electromagnets with Application to the ALS Storage Ring Corrector Magnet Design
}

\author{
Ross D. Schlueter
}

January 28,1991

\begin{abstract}
This memo presents an analytical development for prediction of skew harmonics in a iron core C-magnet to due arbitrarily positioned electromagnet coils. A structured approach is presented for the suppression of an arbitrary number of harmonic components to arbitrarily low values. Application of the analytical harmonic strength calculations coupled to the structured harmonic suppression approach is presented in the context of the design of the ALS storage ring corrector magnets.
\end{abstract}

\section{Summary}

Expressions for skew harmonic components in an [H-, $C-$, or windowframe] electromagnet were analytically derived using a complex varible/conformal mapping technique. They accurately predict the dependence of harmonic component strengths on coil position as verified by experimentally obtained values for the ALS storage ring corrector magnets.

A methodology was developed for the design of electromagnet skew dipoles so that the naturally occurring higher order skew harmonics can be driven arbitrarily close to desired values. This technique was applied to the ALS storage ring corrector magnets, where the quadrupole, sextupole, and octupole components were driven arbitrarily close to zero. Experimentally obtained values for these components were well under the design goal of $1 \%$ of the fundamental for these three harmonic components. Design parameters for the corrector magnets and corresponding harmonic components are tabulated in Figure 8. Tolerancing requirements are summarized in Figure 13.

\section{Introduction}

Harmonics suppression [or control] is often required in accelerator physics magnet applications. For the case of the ALS storage ring corrector magnets, the effect of field errors in the storage ring on the beam's dynamic aperature has been analyzed ${ }^{[1]}$ and resulting harmonics suppression requirements have been tabulated ${ }^{[2]}$. For this corrector magnet application illustrated below, the design goal was that the skew quadrupole, sextupole, and octupole components at $|r|=3 \mathrm{~cm}$ not exceed $1.4 \%, 1.0 \%$, and $1.4 \%$, respectively, of the fundamental. 

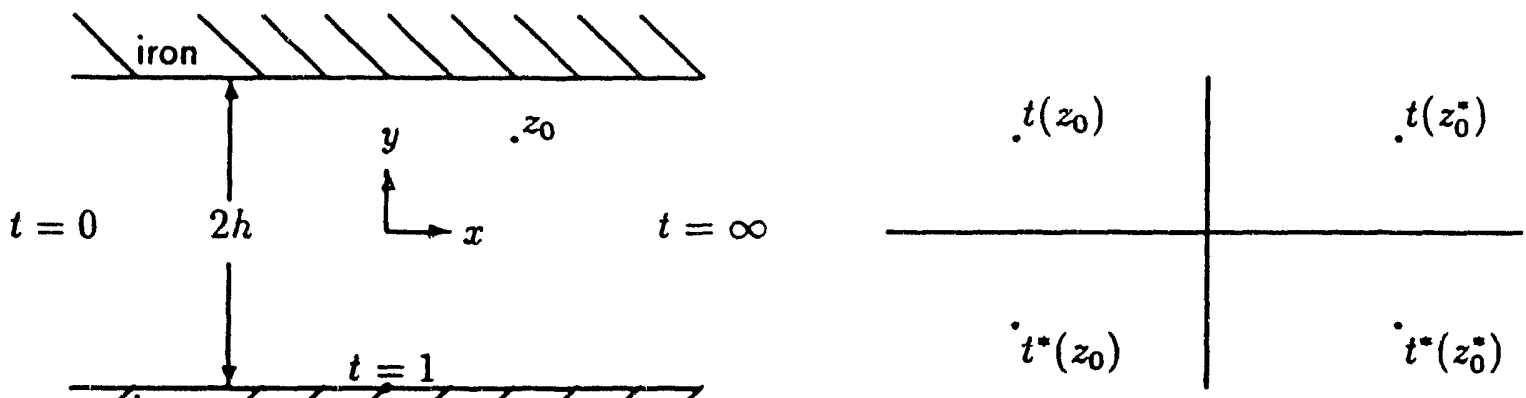

Figure la. Electromagnet dipole $z$ plane geometry

Figure 1b. Schwarz-Christoffel transformed $t$ plane geometry

\section{Analytical Expressions for Skew Harmonic Components in an Electromagnet}

Before we embark on an attempt at harmonics suppression, let us review the formulation of analytical expressions (from Halbach ${ }^{[3]}$ ) for expected skew harmonics in an electromagnet with arbitrarily positioned coils.

Figure 1a shows the gap portion of a wide [x-direction] electromagnet with current filaments of magnitude $\pm I$ at $z_{0}$ and $z_{0}^{*}$, respectively, giving rise to a skew [x-direction] dipole. The return paths for the currents on the outsides of the iron yoke are not shown. The width [x-direction] of the iron pole face yoke is assumed effectively infinite in the analytical model. Exponential decay of field errors as one moves towards the origin from the corner of the iron pole insures that this approximation will not result in large errors of calculated harmonic components near the location of the beam axis (the origin in Figure 1a).

We shall use a Schwarz-Christoffel transformation to conformally map the gap region between $z=(x, \pm h)$ of Figure la to the upper half $t$ plane shown in Figure $1 \mathrm{~b}$. Choosing $t=\infty$ at $z=+\infty$ results in the transformation

$$
\frac{d z}{d t}=\frac{1 / k}{t-a}
$$

Choosing $t=a=0$ at $z=-\infty$ makes $\frac{1}{k}$ take on the value $\frac{2 h}{\pi}$ since at $t=0$, $\operatorname{Im}(\Delta z)=\operatorname{Im}\left(\oint_{t=0} \frac{1}{k t} d t\right), \longrightarrow 2 h=\operatorname{Im}\left(\frac{\pi i}{k}\right)=\frac{\pi}{k}$. Integrating with respect to $t$ gives

$$
z=\frac{2 h}{\pi} \ln t+c, \longrightarrow t=e^{\frac{\pi(x-c)}{2 h}}
$$

Choosing $t=1$ at $z=-i h$ makes $c$ take on the value $-i h$. The complete transformation is thus 


$$
z=\frac{2 h}{\pi} \ln t-i h, \longrightarrow t=i e^{\frac{\pi z}{2 h}}=i e^{k z}=i e^{\alpha}
$$

where $k \equiv \frac{\pi}{2 h}$ and $\alpha \equiv k z$.

For the filament of magnitude $+I$ at $z_{0}$ and its image in the $t$ plane about the real axis, which makes the magnetic field perpendicular to the air-iron interface, the complex potential $F(t)$ is given by

$$
F(t)=\frac{-I}{2 \pi} \ln \left(t-t\left(z_{0}\right)\right)\left(t-t^{*}\left(z_{0}\right)\right)
$$

The magnetic field is related to the complex potential by $H^{*}(z)=i \frac{d F}{d z}$. For the case under consideration we have

$$
H^{*}(z)=i \frac{d F / d t}{d z / d t}=\frac{I k e^{\alpha}}{2 \pi i}\left[\frac{1}{e^{\alpha}-e^{\alpha_{0}}}+\frac{1}{\epsilon^{\alpha}+e^{\alpha_{0}^{\alpha}}}\right]
$$

For current filaments of magnitude $\pm I$ at $z_{0}$ and $z_{0}^{*}$, respectively, and their images in the $t$ plane about the real axis, we have for $H^{*}(z)$ :

$$
H^{*}(z)=i \frac{d F / d t}{d z / d t}=\frac{I k}{2 \pi i} e^{\alpha}\left[\frac{1}{e^{\alpha}-e^{\alpha_{0}}}+\frac{1}{e^{\alpha}+e^{\alpha_{0}^{\alpha}}}+\frac{-1}{e^{\alpha}-e^{\alpha_{0}^{*}}}+\frac{-1}{e^{\alpha}+e^{\alpha_{0}}}\right]
$$

where the relations $t\left(z_{0}\right)=i e^{\alpha_{0}}, t\left(z_{0}^{*}\right)=i e^{\alpha_{0}^{*}}, t^{*}\left(z_{0}\right)=-i e^{\alpha_{0}^{*}}$, and $t^{*}\left(z_{0}^{*}\right)=-i e^{\alpha_{0}}$ have been used. Simplifying, we have

$$
H^{*}(z)=\frac{I k}{2 \pi i}\left[\frac{1}{\operatorname{Sinh}\left(\alpha-\alpha_{0}\right)}-\frac{1}{\operatorname{Sinh}\left(\alpha-\alpha_{0}^{*}\right)}\right]
$$

For a pair of current sheets of magnitude $\pm I^{\prime}$ from $z_{1}$ to $z_{2}$ and from $z_{1}^{*}$ to $z_{2}^{*}$, respectively, and their images in the $t$ plane about the real axis, we have for $H^{*}(z)$ by integrating the terms in Eqn. (7) with respect to $z_{0}$ or $z_{0}^{*}$, as appropriate:

$$
H^{*}(z)=\frac{-I^{\prime}}{2 \pi i}\left[\left.\ln \left(\frac{e^{\alpha_{0}-\alpha}-1}{e^{\alpha_{0}-\alpha}+1}\right)\right|_{\alpha_{0}=\alpha_{1}} ^{\alpha_{0}=\alpha_{2}}-\left.\ln \left(\frac{e^{\alpha_{0}^{*}-\alpha}-1}{e^{\alpha_{0}^{*}-\alpha}+1}\right)\right|_{\alpha_{0}^{*}=\alpha_{i}^{*}} ^{\alpha_{0}^{*}=\alpha_{i}^{*}}\right]
$$

We now decompose these expressions for $H^{*}(z)$ into harmonic components. Define $G(z) \equiv \frac{1}{\operatorname{Sinh}(z)}$. Then,

$$
G\left(\alpha-\alpha_{0}\right)=\frac{1}{\operatorname{Sinh}\left(\alpha-\alpha_{0}\right)}=\Sigma_{0} \alpha^{n} a_{n}=a_{0}+a_{1} \alpha+a_{2} \alpha^{2}+\ldots
$$

where 


$$
n ! a_{n}=\left.\frac{\partial^{n}}{\partial \alpha^{n}}\left(\frac{1}{\operatorname{Sinh}\left(\alpha-\alpha_{0}\right)}\right)\right|_{\alpha=0}=(-1)^{n+1} \frac{\partial^{n}}{\partial \alpha_{0}^{n}}\left(\frac{1}{\operatorname{Sinh}\left(\alpha_{0}\right)}\right)=(-1)^{n+1} G^{n}\left(\alpha_{0}\right)
$$

and where the first equality in Eqn. (10) is true for any function $f\left(\alpha-\alpha_{0}\right)$ that can be expanded in the power series $\Sigma_{0} \alpha^{n} a_{n}$, the second equality in Eqn. (10) is true whenever $f\left(\alpha-\alpha_{0}\right)$ is an odd function, and where the exponent $n$ in $G^{n}\left(\alpha_{0}\right)$ refers to differentiation with respect to $\alpha_{0}$.

Therefore,

$$
\begin{aligned}
G\left(\alpha-\alpha_{0}\right)-G\left(\alpha-\alpha_{0}^{*}\right)= & \Sigma_{0} \alpha^{n}\left(a_{n}-c_{n}\right)=\Sigma_{0} \frac{\alpha^{n}}{n !}(-1)^{n+1}\left\{\frac{\partial^{n} G\left(\alpha_{0}\right)}{\partial \alpha_{0}^{n}}-\frac{\partial^{n} G\left(\alpha_{0}^{*}\right)}{\partial \alpha_{0}^{* n}}\right\}= \\
& \Sigma_{0} \frac{\alpha^{n}}{n !}(-1)^{n+1}\left\{2 i I m\left[\frac{\partial^{n} G\left(\alpha_{0}\right)}{\partial \alpha_{0}^{n}}\right]\right\}
\end{aligned}
$$

Thus, the multipole components for a pair of current filaments of magnitude $\pm I$ at $z_{0}$ and $z_{0}^{*}$, respectively, are given by

$$
\begin{gathered}
H^{*}(z)=\frac{I k}{\pi} \Sigma_{0} \frac{\alpha^{n}}{n !}(-1)^{n+1} \operatorname{Im}\left[\frac{\partial^{n}}{\partial \alpha_{0}^{n}}\left(\frac{1}{\operatorname{Sinh}\left(\alpha_{0}\right)}\right)\right]= \\
\frac{I k}{\pi} \operatorname{Im}\left\{-\frac{1}{S}+\alpha \frac{-C}{S^{2}}-\frac{\alpha^{2}}{2}\left(\frac{1}{S}+\frac{2}{S^{3}}\right)+\frac{\alpha^{3}}{6}(-C)\left(\frac{1}{S^{2}}+\frac{6}{S^{4}}\right)+\ldots\right\}
\end{gathered}
$$

where $S \equiv \operatorname{Sinh}\left(\alpha_{0}\right)$ and $C \equiv \operatorname{Cosh}\left(\alpha_{0}\right)$.

For a pair of current sheets of magnitude $\pm I^{\prime}$ from $z_{1}$ to $z_{2}$ and from $z_{1}^{*}$ to $z_{2}^{*}$, respectively, the multipole components are given by

$$
H^{*}(z)=\frac{I^{\prime}}{\pi} \Sigma_{0} \alpha^{n} \operatorname{Im}\left(k b_{n}\right)
$$

where $\alpha \equiv k z=\frac{\pi z}{2 h}$ and

$$
\begin{gathered}
k b_{0}=-\int_{\alpha_{1}}^{\alpha_{2}} \frac{1}{\operatorname{Sinh}\left(\alpha_{0}\right)} d \alpha_{0}=-\left.\ln \left(\frac{e^{\alpha_{0}}-1}{e^{\alpha_{0}}+1}\right)\right|_{\alpha_{0}=\alpha_{1}} ^{\alpha_{0}=\alpha_{2}} \\
k b_{1}=\left.\frac{1}{\operatorname{Sinh}\left(\alpha_{0}\right)}\right|_{\alpha_{0}=\alpha_{1}} ^{\alpha_{0}=\alpha_{2}}, \quad \cdots \quad, \quad k b_{n}=\left.\frac{(-1)^{n+1}}{n !} \frac{\partial^{n-1}}{\partial \alpha_{0}^{n-1}}\left(\frac{1}{\operatorname{Sinh}\left(\alpha_{0}\right)}\right)\right|_{\alpha_{0}=\alpha_{1}} ^{\alpha_{0}=\alpha_{2}}
\end{gathered}
$$




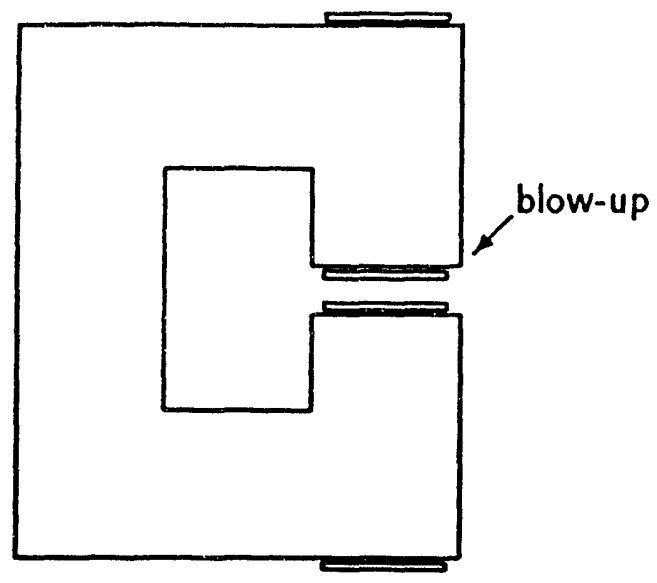

Figure 2a. C-magnet geometry

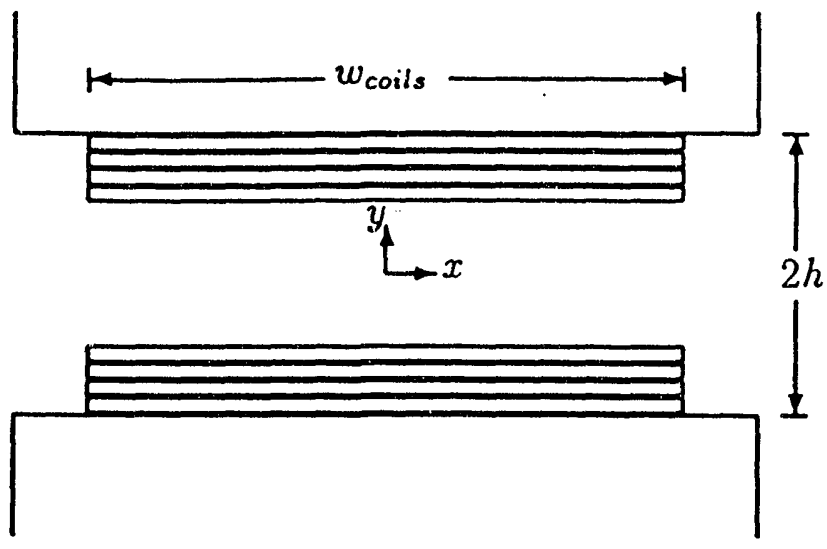

Figure $2 b$. Blow-up of gap region

\section{Electromagnet Coil Design}

\subsection{Design methodology: How to go about a design?}

Judicious selection of coil positions can very effectively reduce unwanted harmonics. An experimental trial and error approach is acceptable for the elimination of one or two harmonics, but this procedure not only becomes more difficult as the number of harmonics to be suppressed increases, but can actually lead one in what at first seems to be the right direction, but is fatally flawed in that it will never converge to a desired solution, (i.e. where the unwanted harmonics can be reduced to an arbitrarily small value). This scenario can arise because the strengths of the harmonic components do not vary linearly with changes in coil position.

A more structured approach to harmonic suppression is possible using the analytical relationships developed in the previous section.

We use as an illustrative example of the design methodoloy, the geometry and specifications for the ALS storage ring corrector magnets (Figure 2). In this example, it is desired to reduce to arbitrarily small values the quadrupole, sextupole, and octupole components; thus a coil arrangement consisting of three independently positionable (in $x$ ) coil packettes is called for.

For the given magnet geometry, ignoring the three-dimensional fringe field effects, errors due to the finite width of the pole face, and the asymmetry due to the $C$-shape, Eqn. (13) may be used to predict how harmonic strengths will vary with coil position for coil packettes of various widths (Figure 3). The various curves in Figure 3 are shown together on a single plot in Figure 4. (Code cmagplot and associated routines, Appendix C, were employed for these computations.)

Determining the harmonics that can be expected from a given coil arrangement consists of superposing the contributions to that harmonic from each of the various coil packettes at their respective locations. 
Figure 3. Skew harnonics strength dependence on coil position

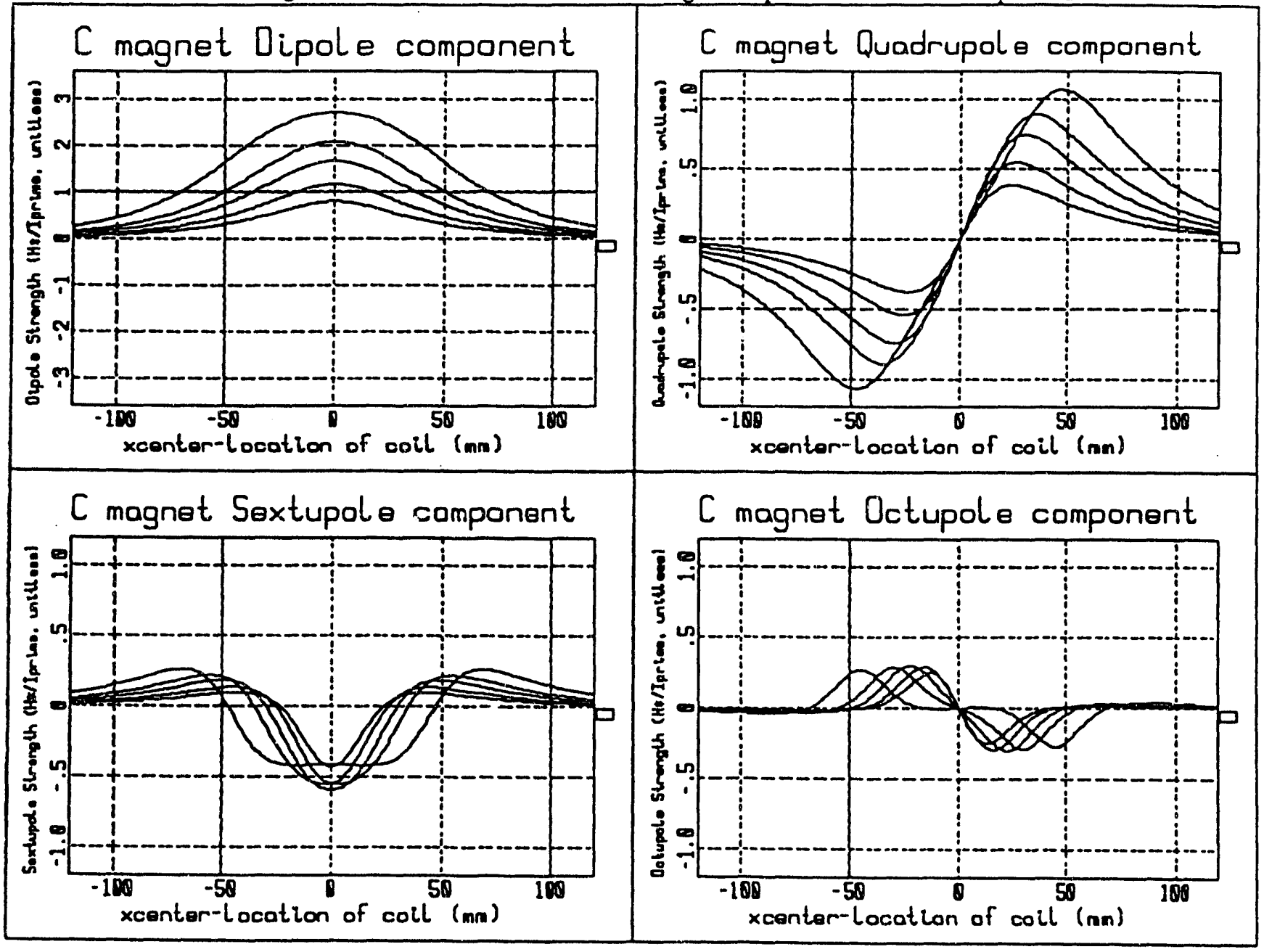

for analytical calculation with $r=30 \mathrm{~mm}, h=57.15 \mathrm{~mm}, y_{\text {coil }}=29.01,37.05,45.09,53.13 \mathrm{~mm}$ curves are for wcoils $=90,60,45,30,20 \mathrm{~mm}$ (widest coil has peaks furthest off-axis) 
Figure 4. Skew harmonic strengths dependence on coil position

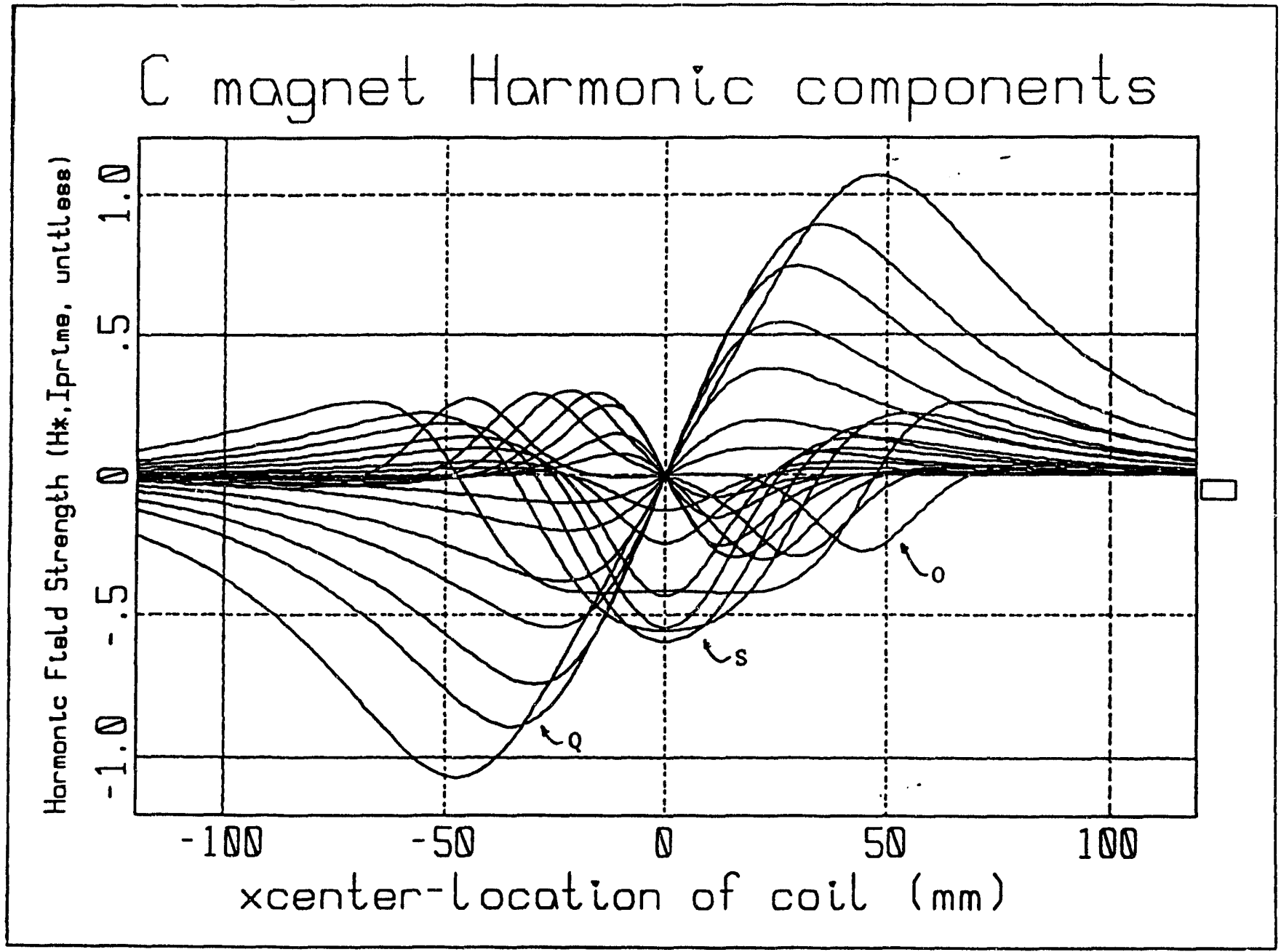

for analytical calculation with $r=30 \mathrm{~mm}, \mathrm{~h}=57.15 \mathrm{~mm}, \mathrm{y}_{\mathrm{colls}}=29.01,37.05,45.09,53.13 \mathrm{~mm}$ curves are for $w_{\text {coil }}=90,60,45,30,20,10,5 \mathrm{~mm}$ (widest coil has peaks furthest off-axis) 
A base arrangement of the coil packettes must first be selected from which the predicted harmonic components may be calculated according to Eqn. (13). Let

$$
x_{i_{\text {base }}}=\text { the base } x \text {-coordinate of the center of coil packette } i \text {, for } i=1,2,3 \text {. }
$$

An $i \times j=3 \times 3$ matrix $[M]$ calculated from the derivatives [with respect to $x$ ] of Eqn. (13) relates changes in the position of our $i(=3)$ coil packettes to changes in the $j(=$ 3) solution vector $\vec{H}^{*}$ consisting of the unwanted quadrupole $(Q)$, sextupole $(S)$, and octupole $(O)$ components:

$$
\vec{H}^{*}=[M] \Delta \vec{x}+\vec{H}_{b a s e}^{*}
$$

where $\vec{H}_{b a s e}^{*}$ are the harmonic strengths when the coil packettes are positioned at their base positions and $\Delta \vec{x}$ are the perturbations of the packettes from those base positions.

A [hopefully improved] guess at a desired coil arrangement is found from inverting the matrix:

$$
\vec{x}_{\text {improved }}=\vec{x}_{\text {base }}+\Delta \vec{x}
$$

where

$$
\Delta \vec{x}=\left[M^{-1}\right]\left(\vec{H}_{\text {desired }}^{*}-\vec{H}_{\text {base }}^{*}\right)
$$

Iteration is necessary due to the nonlinearity of $[M]$ over the finite calculated changes in coil packette position $\Delta \vec{x}$. Several points are worth noting here:

- The calculated direction of movement may head toward a local maximum/minimum (i.e. a 'hump') in which case a converged solution may not be possible for this chosen starting point (i.e. the chosen $x_{i_{\text {bare }}}, i=1,2,3$ ). However, the curves in Figure 4 will indicate if this is the case, thus avoiding a futile experimental search.

- There may be no solution possible for a given chosen set of coil packette widths no matter what starting point is chosen. Again, the curves in Figure 4 will indicate if this is the case, and they can be used to select a set of coil packette widths for which a solution does exist.

- Operating near a 'hump' is not categorically to be avoided; only so for the case where one is depending on the perturbation of that particular pacrette to be able to significantly change that particular harmonic. Again, the curves in Figure 4 can be used to enable a judicious selection of packette sizes and base positions.

- One must insure that variations in the elements of $[M]$ over the possible excursions of the packettes from their base positions will not cause the the matrix to become singular, and thus the problem as given, intractable. Appendix A discusses experimental uncertainties and variation of matrix elements as they relate to the condition of matrix $[M]$. 
In summary, the key to the magnetics design is to use the general harmonic curves (Figure 4) to superpose for each unwanted harmonic the contributions from a combination of suitablly sized coil packettes located at suitable base positions. Iteration on a solution to reduce unwanted harmonics to an arbitrarily low value is straightforward; experimentally we were able to reduce the $Q, S$, and $O$ components for the ALS storage ring corrector magnets to below $\frac{1}{4}$ of $1 \%$ of the skew dipole in just two iterations.

\subsection{Application to ALS Storage Ring Corrector Magnets}

The following discussion retraces the design thought process for the ALS storage ring corrector magnets and serves as a guide for any similar future design involving specification of harmonic components.

\subsubsection{Prototype Coil Design}

Experimental results of harmonics strength dependence on prototype coil packette position for coil packettes of various widths are shown in Figure 5. A comparison of Figure 5 with its two-dimensional analytical counterpart, Figure 4, confirms that the analytical Eqn. (13) models the observed harmonic behavior very well. That this is so, illustrates the exponential decay of field errors as one moves inside the magnet from the corner of the iron pole. Minor differences between the two graphs is attributable to the asymmetry of the $C$-shape, the finite width of the pole face, and the three-dimensional fringe fields. The most salient asymmetry effect (see Figure 5) is that the positive and negative peaks of the quadrupole plots differ in magntitude $~ 17 \%$.

The coil packette positions for $C$-magnet prototype tests were selected as set forth below:

- A total of 24 turns per layer in each of four layers was chosen. For the individual prototype coil width of $7.85 \mathrm{~mm} / \mathrm{turn}$, the total wound prototype coil width is 188.4 $\mathrm{mm}$, which fits within the alloted $220 \mathrm{~mm}$ mold gap supporting the coils, leaving $\sim 30 \mathrm{~mm}$ of 'play' for coil positioning.

- Three indpendently pósitionable coil packettes were chosen so as to be able to reduce to arbitrarily small values the three unwanted harmonic components: quadrupole, sextupole, and octupole.

- So as to keep from having to move too far out from the center position to null the quadrupole component, which would result in a decrease in magnitude of the fundamental, the 24 coils were inititially split 13/11, with 13 coils going on the side with the smailer magnitude quadrupole peak (i.e. negative $x$ in Figure 5).

- Looking for a base position that would result in relatively low $(\sim 1 \%)$ net $Q, S$, and $O$, while simultaneously positioning for good matrix properties, the 24 coils were split $13 / 3 / 8$ into three packettes, centered at $x=-55.0 \mathrm{~mm}, 23.6 \mathrm{~mm}$, and 66.8 $\mathrm{mm}$, respectively. This base position features a well-conditioned matrix $[M]$ whose elements have units of ' $\frac{H^{*}}{I^{\prime}} / \mathrm{mm}$ packette displacement from base position': 
Figure 5. Skew harmonic strengths dependence on coil position

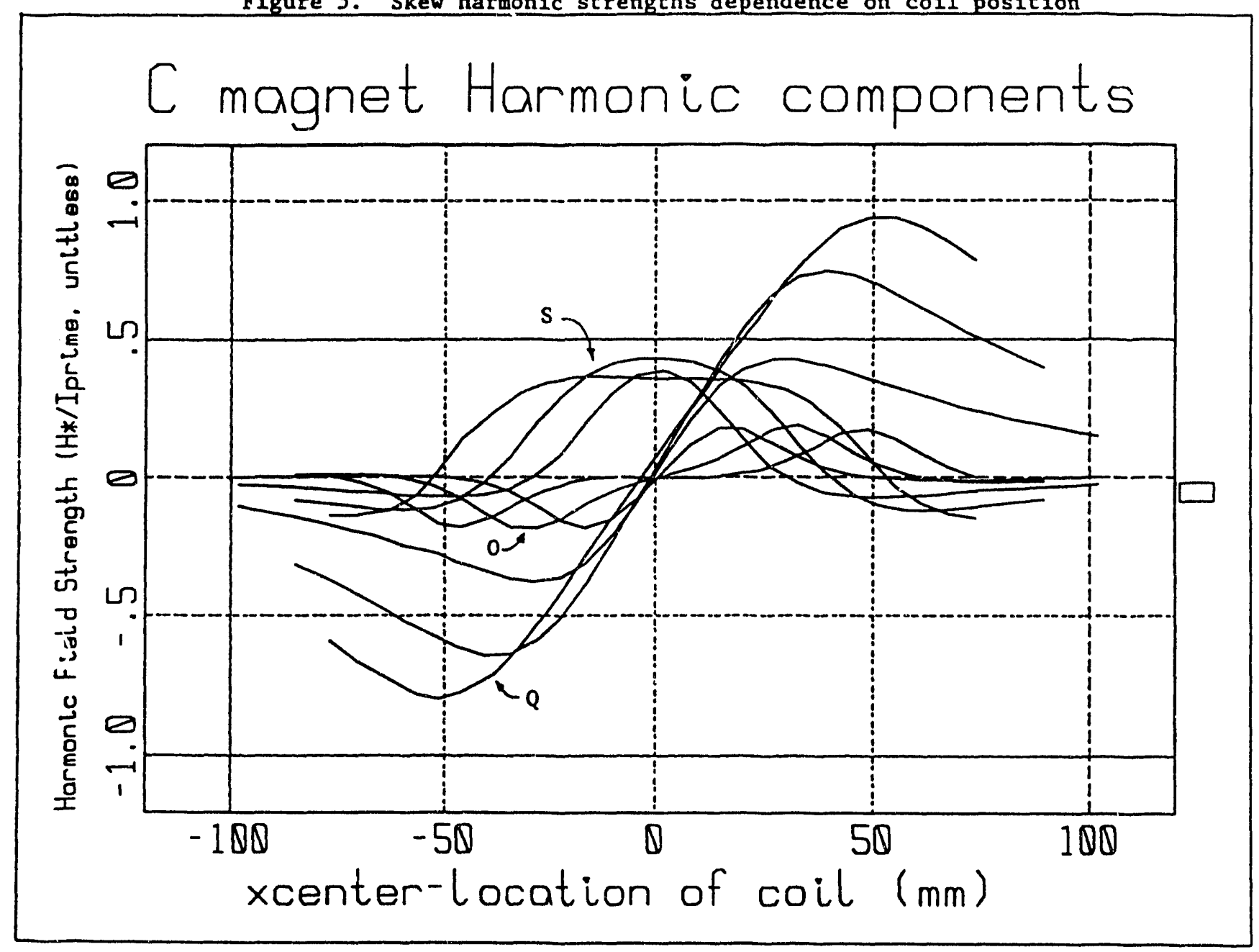

for experimental data with $r=30 \mathrm{~mm}, \mathrm{~h}=57.15 \mathrm{~mm}, \mathrm{y}_{\text {coils }}=29.01,37.05,45.09,53.13 \mathrm{~mm}$ curves are for $w_{\text {coils }}=94.20,62.80,31.40 \mathrm{~mm}(12,8,4$ turns), (widest coil has peaks furthest off-axis) 


$$
M=\left(\begin{array}{rrr}
-0.005 & 0.006 & -0.0075 \\
0.013 & -0.014 & 0.0002 \\
-0.007 & -0.009 & -0.0004
\end{array}\right)
$$

and which will not become singular as its elements vary with the perturbations of coil packette positions necessary to drive to zero the unwanted harmonic components (See Appendix A.) Units of $[M]$ can be converted to $\%$ of dipoie/mm packette displacement from base position', using the normalized dipole strength of ' 4.0 '. Note that this base position has packettes \#2 and \#3 abutting. However, since the estimated net quadrupole component for the base position is positive, (not by accident), we expect the $3^{\text {rd }}$ packette will need to be moved in the positive direction from its base position to null the quadrupole term. The simultaneous change in the sextupole component will be minimal since the $M_{3,2}$ element is small. Since the $M_{2,2}$ element is large, a smaller perturbation of the position of the $2^{\text {nd }}$ packette will make sextupole corrections without overlapping the physical space occupied by the $3^{\text {rd }}$ packette. The experimentally obtained values for the $Q, S$, and $O$ at this base coil position were $+1.6 \%,-1.5 \%$, and $-1.0 \%$, respectively.

- The packette base positions and corresponding experimentally obtained values for the $Q, S$, and $O$ along with the matrix $[M]$ are input into a matrix solver code cmagmate (see Appendix $C$ ) which inverts the matrix $[M]$ and performs the operations given by Eqns. (15) and (16) to give a predicted positioning of the packettes which will null the $Q, S$, and $O$ components.

- The output configuration is experimentally tested and the three componets are brought to $\leq 0.25 \%$ of the fundamental in just two iterations. The final configuration comprises packettes centered at $-56.6 \mathrm{~mm}, 18.6 \mathrm{~mm}$, and $71.9 \mathrm{~mm}$, respectively, yielding experimentally obtained values for the $Q, S$, and $O$ of $-0.02 \%,-0.13 \%$, and $-0.10 \%$, respectively.

- The same procedure was used to null unwanted harmonics for the case when adjacent quadruplole cores, which alter the harmonic components, are present.

\subsubsection{Production Coil Design}

With the analytical design tools and experimental design strategy proven on prototype coils, a second (smaller) set of experimental runs was required using production coils, whose dimensions differed from the prototype coils. The 24 coil prototype design with packette positions as recorded above, used with production coils would give $Q, S$, and $O$ components $\sim 1-2 \%$ of the dipole. This is because the production coil cross section (width $=6.86 \mathrm{~mm}$ ) with thinner (and less robust) insulation, is smaller than that of the prototype coils. The shorter width coil packettes give rise to slightly different harmonic component contributions. Also, the off-axis position of the 4 coil layers is different; thus a new set of generic 'harmonic strengths versus coil position' plots (Figure 6) are required for the production coil $y$-positions. The various curves in Figure 6 are shown together on a single plot in Figure 7. A comparison of Figures 4 and 7 reveals that for a given length coil packette, the small change in packette off-axis $y$-position results in a change in peak magnitude of the harmonics but a change of the $x$-position of the peaks is only barely 
Figure 6. Skew harmonics strength dependence on coil position

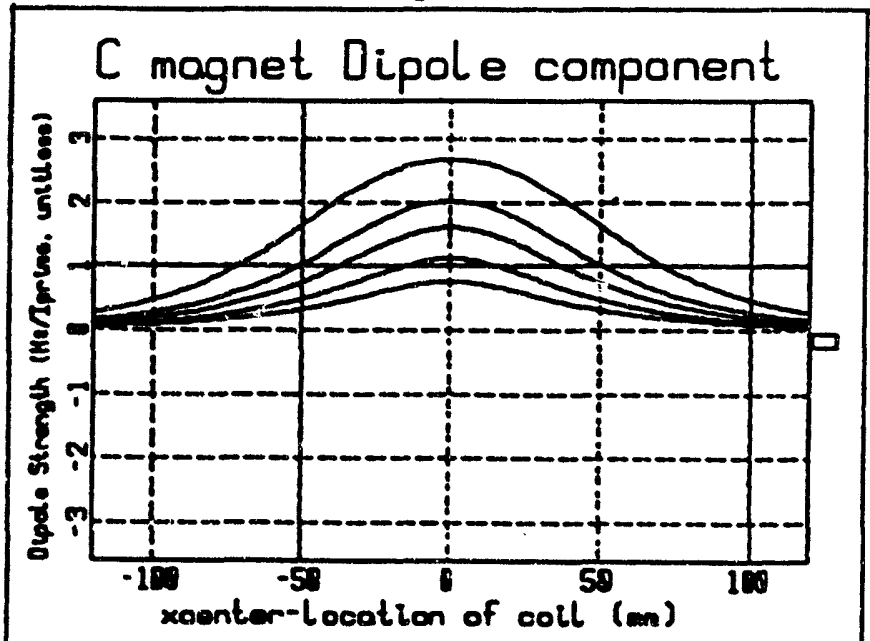

C magnet Quadrupole component

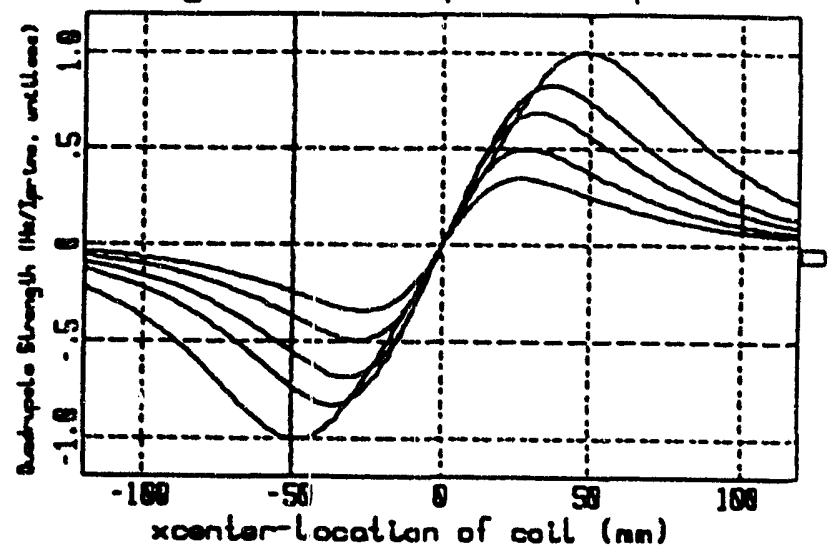

C magnet Sextupole component

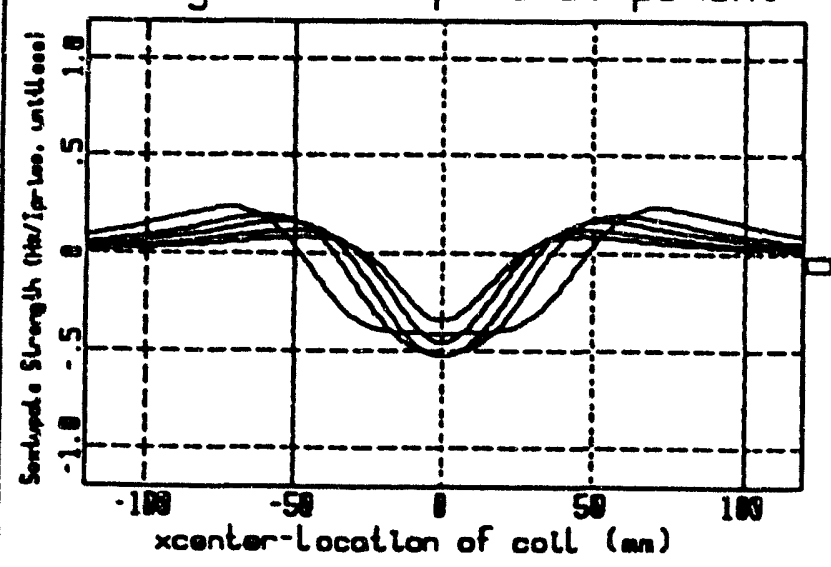

C magnet Detupole component

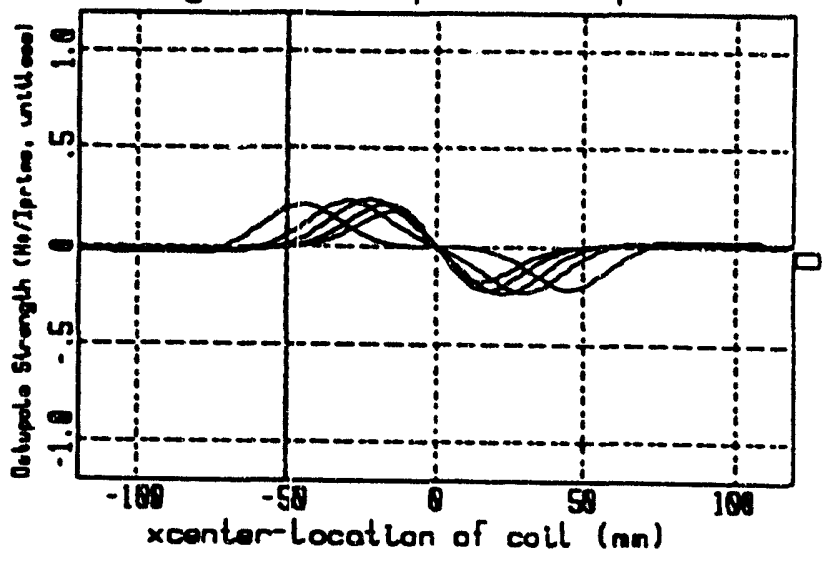

for analytical calculation $\mathrm{wth} r=30 \mathrm{~m}, \mathrm{~h}=57.15 \mathrm{~mm}, y_{\text {colls }}=33.59,40.32,47.05,53.78 \mathrm{~mm}$ curves are for "co1ls $m 90,60,45,30,20 \mathrm{~m}$ (widest coll has peaks furthest off-axis) 
Figure 7. Skew harmonics strength dependence on coil position

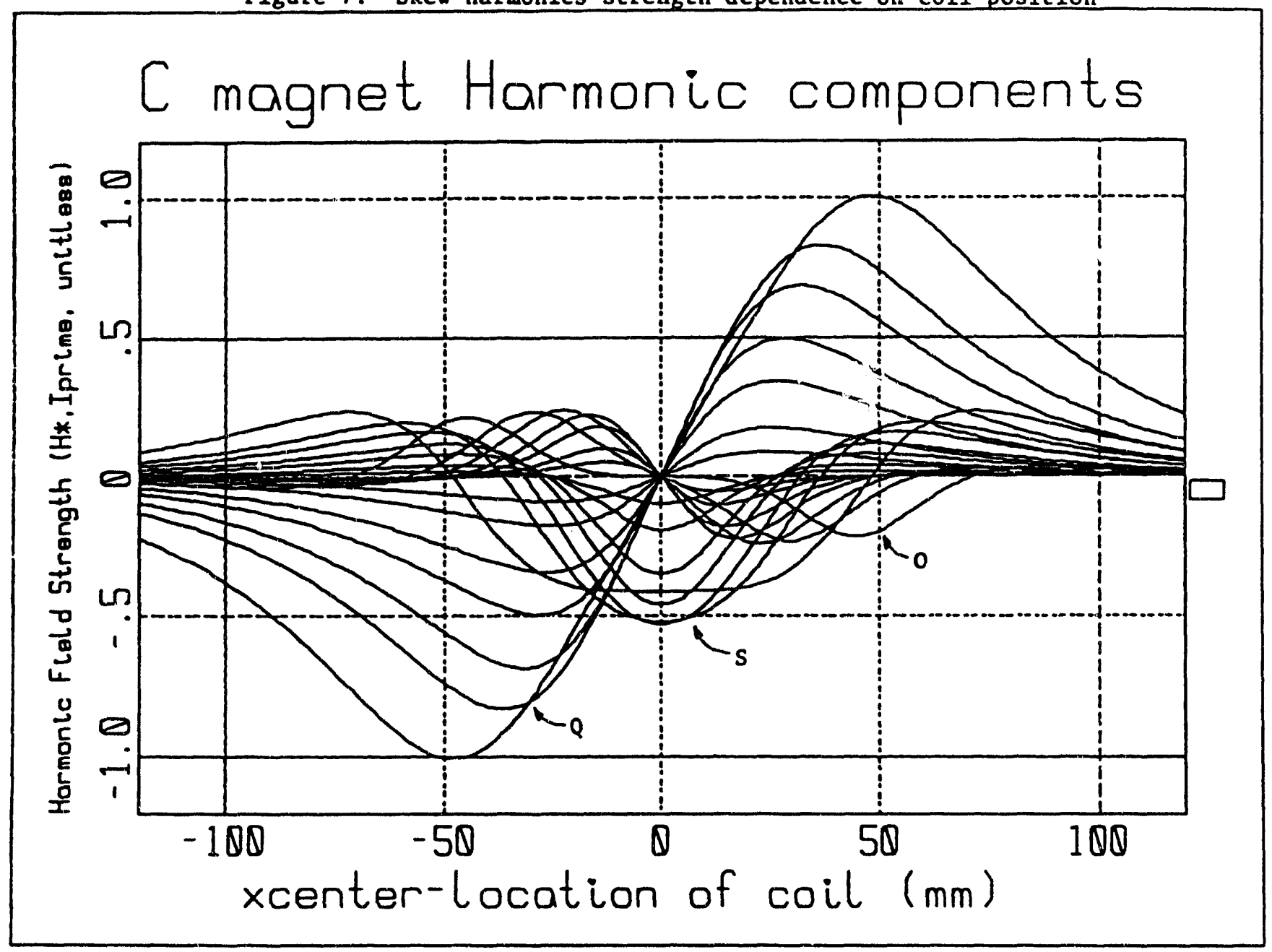

for analytical calculation with $r=30 \mathrm{~mm}, h=57.15 \mathrm{~mm}, y_{\text {coils }}=33.59,40.32,47.05,53.78 \mathrm{~mm}$ curves are for $w_{c 011 s}=90,60,45,30,20,10,5 \mathrm{~mm}$ (widest coll has peaks furthest off-axis) 
noticeable. Thus we used the prototype coil experimental curves of Figure 5 (interpolated for appropriate production coil packette [ $x$-direction] widths) as an aide in determining the matrix elements and suitable base positions for our production coil packette arrangement.

The coil packette positions for $C$-magnet tests with production coils were selected via a procedure analogous to that of the previous section for the prototype coils, with the following resultant design:

The coil arrangement consists of a total of 25 turns per layer in each of four layers, resulting in a total wound prototype coil width of $171.5 \mathrm{~mm}$, which fits within the alloted $220 \mathrm{~mm}$ mold gap supporting the coils, leaving $\sim 50 \mathrm{~mm}$ of 'play' for coil positioning. The coils were split $13 / 3 / 9$ into three packettes, centered at $-54.0 \mathrm{~mm}, 15.7 \mathrm{~mm}$, and $69.3 \mathrm{~mm}$. The corresponding well-conditioned matrix for this arrangement is (in units of ' $\frac{H^{*}}{I^{\prime}} / \mathrm{mm}$ packette displacement from base position'):

$$
M=\left(\begin{array}{rrr}
-0.006 & 0.006 & -0.0075 \\
0.013 & -0.013 & 0.0010 \\
-0.008 & -0.004 & 0.0000
\end{array}\right)
$$

Units of $[M]$ can be converted to $\%$ of dipole/mm packette displacement from base position', using the normalized dipole strength of ' $4.0^{\prime}$ '. The experimentally obtained values for the $Q, S$, and $O$ for this optimal isolated coil configuration \#9 are $+0.11 \%$, $+0.04 \%$, and $-0.16 \%$, respectively. An optimal configuration \#10 for the case when two adjacent quadruplole cores are present on either end of the $C$-magnet (each having 6" iron-iron distance), comprises packettes centered at $-52.0 \mathrm{~mm}, 20.2 \mathrm{~mm}$, and 73.7 $\mathrm{mm}$, respectively, yielding experimentally obtained values for the $Q, S$, and $O$ of $-0.08 \%$, $-0.38 \%$, and $-0.23 \%$, respectively.

A compromise single configuration \#7 giving the best overall results when used in both the isolated position and the position with two adjacent quadrupole cores comprises packettes centered at $-52.1 \mathrm{~mm}, 17.6 \mathrm{~mm}$, and $70.7 \mathrm{~mm}$, respectively, yielding experimentally obtained values for the $Q, S$, and $O$ of $-0.04 \%,+0.09 \%$, and $+0.60 \%$, respecti ly in the isolated position and $+0.19 \%,+0.66 \%$, and $+0.29 \%$, respectively in the position with adjacent quadrupole cores.

These results, along with those for the 10-, 12-, and 14-pole components are tabulated in Figure 8.

\subsection{3 $C$-magnet Positions and Environment in the Storage Ring}

In a typical storage ring section comprising $\frac{1}{12^{\text {th }}}$ of the ring, there are four corrector magnets. The first is neighbored by an upstream undulator end clamp (7.4" iron-iron distance) and a downstream quadrupole (13" iron-iron distance). The second and third corrector magnets are neighbored by upstream and downstream quadrupoles, both making a 6" iron-iron distance with the corrector magnets. The fourth is neighbored by an upstream quadrupole (19" iron-iron distance) and a downstream undulator end clamp (15" iron-iron distance). 
Figure 8. Experimental multipole strength data for candidate production coil configurations

\begin{tabular}{|l|l|r|r|r|}
\hline & configuration: & $\# 9$ & $\# 10$ & $\# 7$ \\
\hline packette & $x_{c_{1}}$ (mm): & -54.0 & -52.0 & -52.1 \\
center & $x_{c_{2}}(\mathrm{~mm}):$ & 15.7 & 20.2 & 17.6 \\
position: & $x_{c_{3}}$ (mm): & 69.3 & 73.7 & 70.7 \\
\hline & & & & \\
isolated: & & & & \\
& quadrupole: & 0.11 & -0.22 & 0.04 \\
& sextupole: & 0.04 & -0.57 & 0.09 \\
\% of & octupole: & -0.16 & -0.97 & -0.60 \\
dipole & & & & \\
& 10-pole: & -1.86 & -2.29 & -2.00 \\
& 12-pole: & 0.69 & -0.12 & 0.27 \\
& 14-pole: & -1.18 & -1.28 & -1.25 \\
& & & & \\
\hline w/ adjacent & quadrupole: & 0.30 & -0.08 & 0.19 \\
quad cores: & sextupole: & 0.57 & -0.38 & 0.66 \\
\% of & octupole: & 0.81 & -0.23 & 0.29 \\
dipole & & & & \\
& 10-pole: & -2.17 & -2.75 & -2.35 \\
& 12 -pole: & 0.92 & -0.14 & 0.35 \\
& 14-pole: & -1.63 & -1.78 & -1.72 \\
\hline
\end{tabular}




\begin{tabular}{|l|r|r|r||r||r|r|}
\hline environment: & isolated & $\begin{array}{r}\text { a plate } \\
\text { at }-7.4^{\prime \prime}\end{array}$ & $\begin{array}{r}\text { a quad } \\
\text { at }+13^{\prime \prime}\end{array}$ & $\begin{array}{r}2 \text { quads } \\
\text { at } \pm 6 "\end{array}$ & $\begin{array}{r}\text { a plate } \\
\text { at }+15^{\prime \prime}\end{array}$ & $\begin{array}{l}\text { plate at }+15^{\prime \prime} \\
\text { quad at }-19^{\prime \prime}\end{array}$ \\
\hline dipole $\left(\frac{H^{*}}{L^{\prime}}\right):$ & 3.70 & 3.45 & 3.63 & 2.82 & 3.53 & 3.60 \\
& & & & & & \\
quadrupole (\% dipole): & 0.04 & 0.03 & 0.02 & 0.19 & -0.20 & -0.14 \\
sextupole (\% dipole): & 0.09 & 0.14 & 0.11 & 0.66 & 0.07 & 0.11 \\
octupole (\% dipole): & -0.60 & -0.64 & -0.61 & 0.29 & -0.63 & -0.63 \\
\hline
\end{tabular}

Figure 9. Multipole strength data for coil configuration \#7 in various environments

For the middle two corrector magnets, experimental magnetics designs were tested simulating their environment when positioned in the storage ring, i.e. with the adjacent quadrupole cores in position. For the first and fourth corrector magnets, experimental magnetics designs were tested in an isolated environment, i.e. with no other ferromagnetic hardware nearby. In Figure 9 harmonic components are tabulated for the configuration \# 7 design in a variety of environments to verify that the first and fourth corrector magnets are effectively isolated.

The end clamp even at she close distance of -7.4 " has virtually no effect on the harmonics components. It should be noted that the sampling area of the rotating coil of the experimental measurement system extends to $\pm 13^{\prime \prime}$ beyond the ends of the $C$-magnet iron. Thus near on-axis field contributions beyond these $z$ locations are not measured. Harmonic components this far out in $z$ should be small, however. In any event, the effect of an adjacent quadrupole at either $+13^{\prime \prime}$ or -19 " lies somewhere between that of the two experimentally tested environments of Figure 8 , for which configuration \#7 is a suitable magnetics design.

\subsubsection{Tolerances}

Figure 10 shows a cross section of the configuration \#7 coil design. Assume the largest acceptable uncertainty in harmonic component strengths in the final coil design is $0.25 \%$ of the fundamental for all harmonics. (This is $\leq \frac{1}{4}$ of the target magnitudes of $\sim 1 \%$ for each of the three harmonics, $Q, S$, and $O$.) Here we investigate the harmonic component errors introduced by deviations of dimensions from the ideals and the implications for dimensional tolerances. 'Ideal' is understood to mean the actual experimental configuration \#7 dimensions that resulted in the suppression of harmonics as given in Figure 8.

$x$-positioning Displacements. The effect of a millimeter displacement in the $x$ direction of one of the three coil packettes on the $Q, S$ and $O$ harmonics is given directly by the elements of the matrix $M$ of Eqn. (18).

The effect of a deviation from ideal of a coil packette width is illustrated by the pairs of curves in Figure 11, where harmonic components are plotted for both the design widths for the three coil packettes and for small perturbations of those widths from the ideal. 


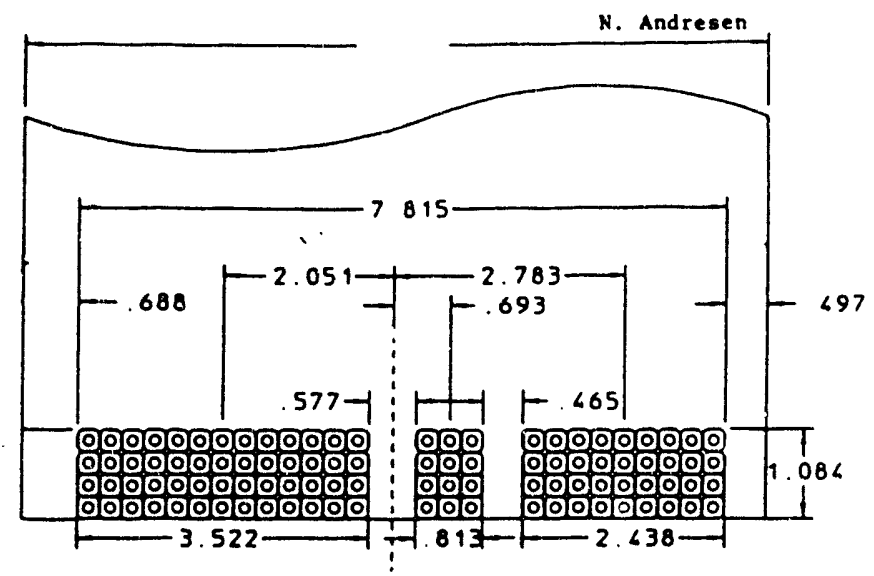

Figure 10. Cross section of the configuration \#7 coil design

y-positioning Displacements. In the $y$ direction the coils are stacked from the iron outward. The effect of a small stack up displacement in the $y$ direction of the coils furthest from the iron of one of the three coil packettes on the $Q, S$ and $O$ harmonics is illustrated by the pairs of curves in Figure 12, where the harmonic components are plotted for both the design $y_{c}$ positions and for small perturbations of those positions from the ideal.

$z$-positioning Displacements. (Here $z$ is the coordinate in the axial direction, rather than the complex variable $z=x+i y$ used in the analytical sections). Experimental results for $z$-position displacements of the coil packettes with respect to the iron core showed virtually no effect on harmonic components over the range of coil movement possible ( $t$ $\left..050^{\prime \prime}\right)$.

Tolerancing Implications. In Figure 13 harmonics contributions caused by $+1 \mathrm{~mm} x$, $y$, and $z$ positioning errors and coil width errors are tabulated. The toial positioning errors error budget ( $0.25 \%$ of dipole for each of the harmonics components) is allocated so as to distribute the mechanical assembly level of difficulty uniformly. Resulting tolerances required to meet the error budget for each of the harmonics is tabulated. For the coil packette width error, two scenarios are investigated: leaving the packette center positions $x_{c}$, unchanged and leaving the packette inside edge position unchanged. The latter proves to be the desired approach, as it increases the dimensional requirement of the $w_{c}$ tolerance and it replaces the sextupole with the quadrupole as the component that sets the tolerance on coil packette width. The important error figures and suggested design tolerances are found in columns $e, p, u$, and $v$ for $x_{c}, w_{c}, y_{c}$ and $z_{c}$, respectively. The sextupole-limited coil packette $x_{c}$ position tolerance of .009" (column e) assumes that $x_{c}$ positionings of the three coil packettes are independent, and do not translate as a unit. The quadrupole-limited allowable coil width $w_{c}$ variation of .012" (column p) for the second coil packette (having three turns) assumes that the three coil packette widths per ideal width will be uniformly at variance from the ideal and that the design approach of making the inner $x$-positions of the coil packettes invariant, rather than the $x_{c}$ coil center positions, is employed. Tolerances on $w_{c}$ for the two larger coil packettes scales proportionately with their respective $x$-direction widths. The sextupole-limited allowable coil $y_{c}$ position variation of .012" (column $u$ ) assumes that the three coil packettes $y_{c}$ positions will be uniformly at variance from the ideal. Harmonics errors introduced by $z_{c}$ 
Figure 11. Effect of a deviation from the ideal of a coil packette width

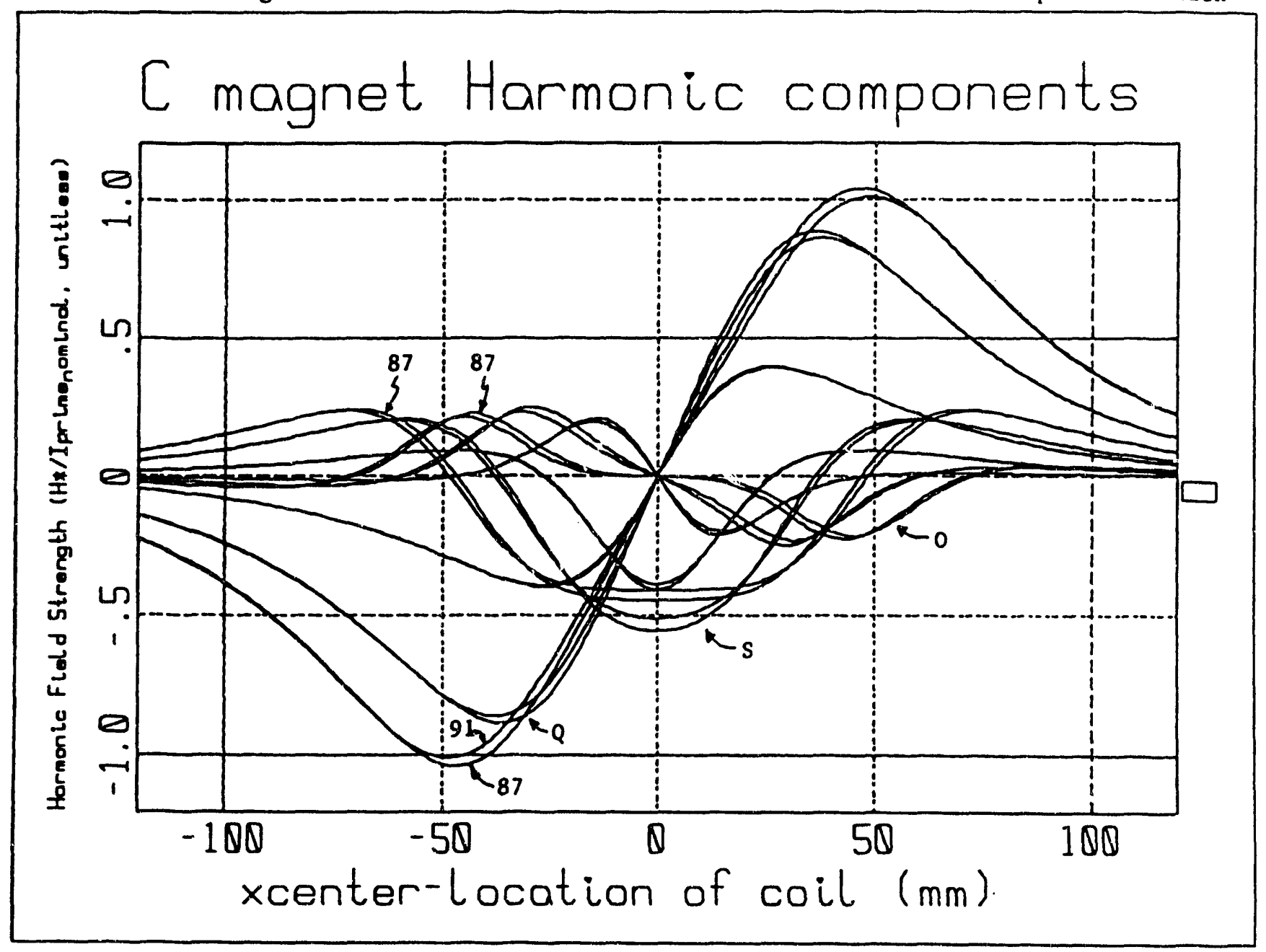

for analytical calculation with $r=30 \mathrm{~mm}, h=57.15 \mathrm{~mm}, y_{\text {colls }}=33.59,40.32,47.05,53.78 \mathrm{~mm}$ curves are for $w_{c o i l s}=91$ vs. 87,64 vs. 60,23 .vs. $19 \mathrm{~mm}$ (widest coil has peaks furthest off-axis) 
Figure 12. Effect of a deviation from the ideal of coil "y" potition

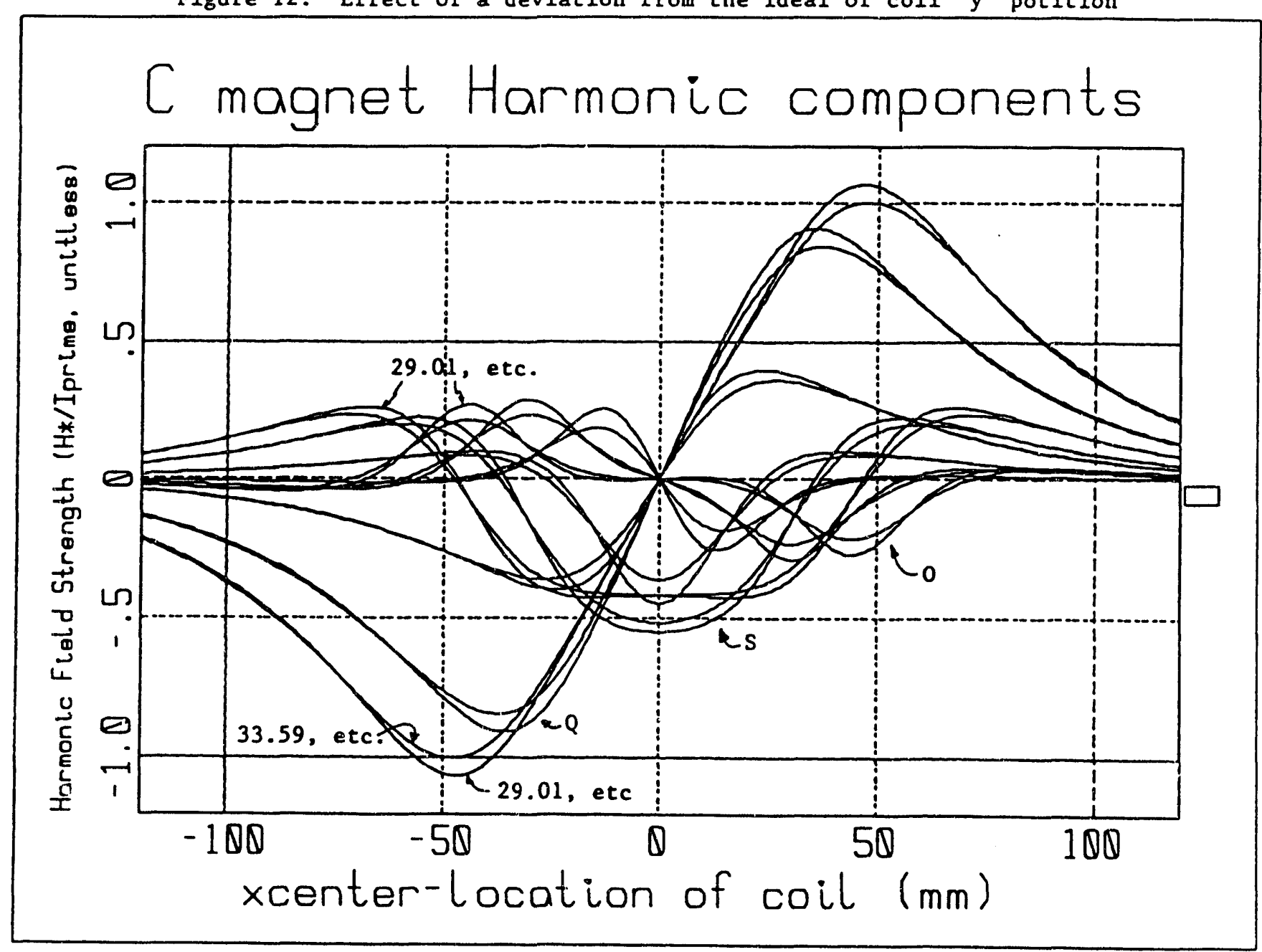

for analytical calculation with $r=30 \mathrm{~mm}, h=57.15 \mathrm{~mm}, w_{c o i l s}=89,62,21 \mathrm{~mm}$ curves are for $y_{c 011 s}=29.01,37.05,45.09,53.13$ vs. 33.59, 40.32, 47.05, 53.78 mm 


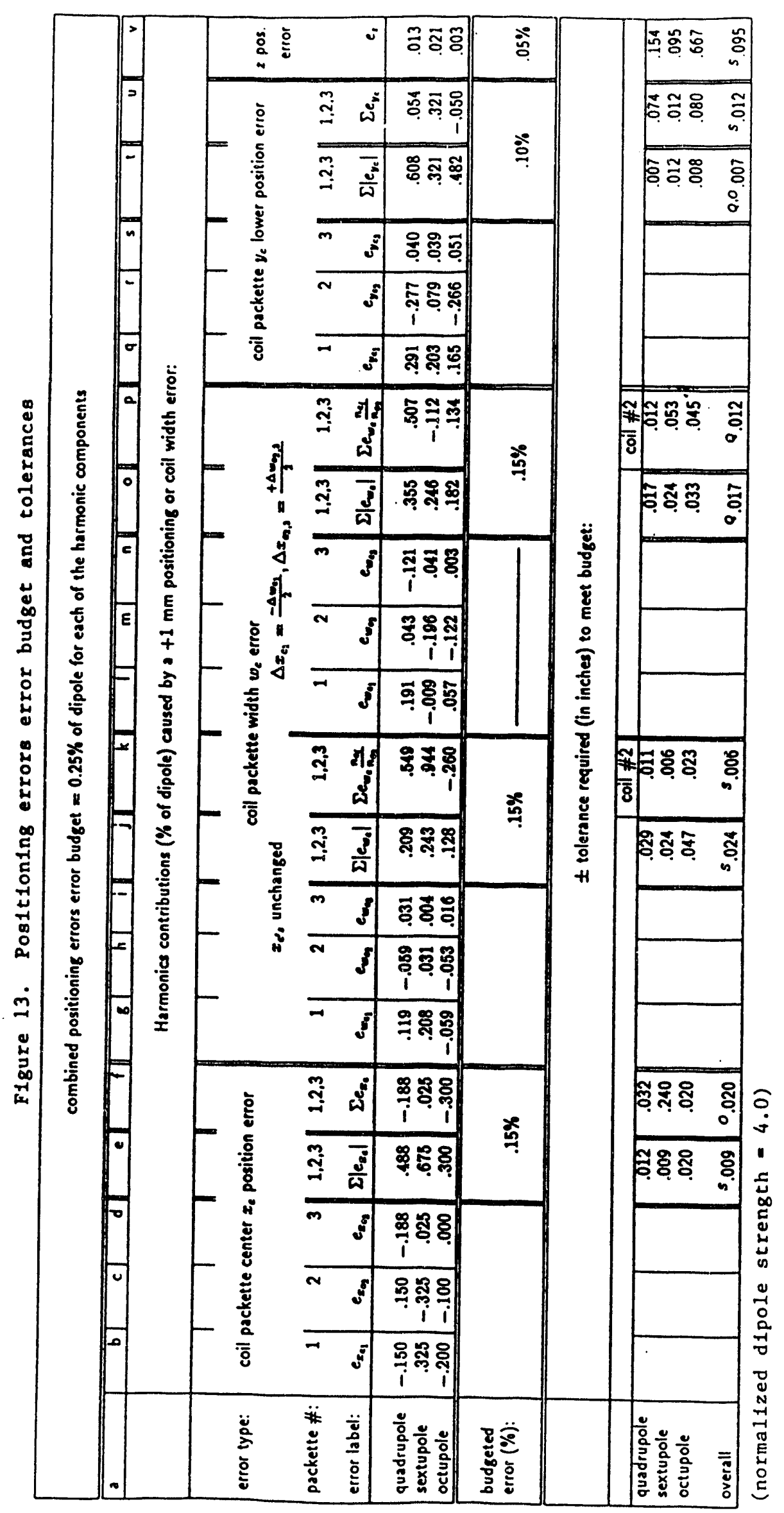


position errors (column v) are not significant.

All \% error values in the above figure are normalized with respect to a dipole having a normalized magnitude $\frac{H^{*}}{l^{\circ}}=4$. Actual normalized dipole strength ranges from 2.8 to 3.7 . depending on the corrector magnet's environment (see Figure 9). Thus actual tolerances required are $70-90 \%$ of those listed in Figure 13.

Rotating Coil Measurement System Alignment Requirement. As seen in Figure 8 , the configuration \#7 design suppresses the on-axis harmonics $a_{n}$ given by $H^{*}(z)=\sum_{n=0} a_{n} z^{n}$ to well below $1 \%$ of $a_{0}$ for $n=1,2,3$ and well below $3 \%$ of $a_{0}$ for $n=4,5,6$ as measured by a rotating coil measurement system which defines the on-axis position. If, in the performance testing of subsequent magnets, the rotating coil is off-axis by an ariount $\Delta_{z}$ such that $w=z-\Delta_{z}$, then the measured coefficients $b_{n}$ defined by $H^{*}(w)=\sum_{n=0} b_{n} w^{n}$ are related to the on-axis coefficients by expanding $H^{*}(z)$ in $w$ :

$H^{*}(z)=\Sigma_{n=0} a_{n} z^{n}=\Sigma_{n=0} a_{n}\left(w+\Delta_{z}\right)^{n}=\Sigma_{n=0}\left\{w^{n} \Sigma_{m=n}\left(\frac{a_{m} m ! \Delta_{z}^{m-n}}{(m-n) ! n !}\right)\right\} \equiv \Sigma_{n=0} w^{n} b_{n}$

The $b_{n^{\prime}}$ can be thought of as measured values of the $a_{n^{\prime}}$ that are in error due to the $\Delta_{z}$ measurement coil misalignment. We budget for an $f_{n} \%$ of $a_{0}$ at $|r|=3 \mathrm{~cm}$ measurement coil misalignment-induced error for the $n^{\text {th }}$ harmonic (i.e. $r^{n}\left|b_{n}-a_{n}\right| \leq f_{n} a_{0}$ ). Then, defining $\epsilon \equiv \frac{\Delta_{z}}{r}$ and $p_{n} \equiv \frac{a_{n} r^{n}}{a_{0}}$, we have

$$
f_{n} \geq \frac{r^{n}}{a_{0}}\left|b_{n}-a_{n}\right|=\frac{r^{n}}{a_{0}} \Sigma_{m=n+1}\left(\frac{a_{m} m ! \Delta_{z}^{m-n}}{(m-n) ! n !}\right)=\Sigma_{m=n+1}\left(\frac{m ! p_{m} \epsilon^{m-n}}{(m-n) ! n !}\right)
$$

For the storage ring corrector magnets, at $|r|=3 \mathrm{~cm}, p_{n} \leq .01$ for $n=1,2,3$, $p_{n} \leq .03$ for $n=4,5,6$ and $p_{n} \simeq 0$ for $n>6$. Thus if $f_{n}=0.2 \%=.002$ for $n=1,2,3$ the maximum allowable $\epsilon=.016 \Longrightarrow \Delta_{z} \leq .048 \mathrm{~cm}\left(.019^{\prime \prime}\right)$, at which measurement coil misalignment-induced errors of $Q, S$, and $O$ are $.03 \%, .05 \%$, and $.20 \%$ of the dipole, respectively. If we choose to keep $f_{n}=.0012$ for $n=1,2,3$ the maximum allowable $\epsilon=.016 \Longrightarrow \Delta_{z} \leq .030 \mathrm{~cm}\left(.012^{\prime \prime}\right)$, at which measurement coil misatignment-induced errors of $Q, S$, and $O$ are $.02 \%, .03 \%$, and $.12 \%$ of the dipole, respectively.

\subsubsection{Subtleties and Refinements}

A Subtlety. Normalization of the three-dimensional magnetic field experimental data for comparison with field magnitudes in the two-dimensional analytical model is not straightforward. Use and derivation of an effective coil length in the axial direction $L_{\text {eff }}$ for the experimental data is discussed in Appendix $B$.

A Refinement. Figure 14 is effectively a continuation of Figure 6 . It shows analytical curves for two-dimensional '10-, 12-, and 14-pole strengths versus prototype coil packette position' plots. Figure 15 shows the analogous experimentally obtained plots for these harmonics. The accuracy of the analytical plots even for these higher harmonics is striking! 
Figure 14. Skew harmonics strength dependence on coil position

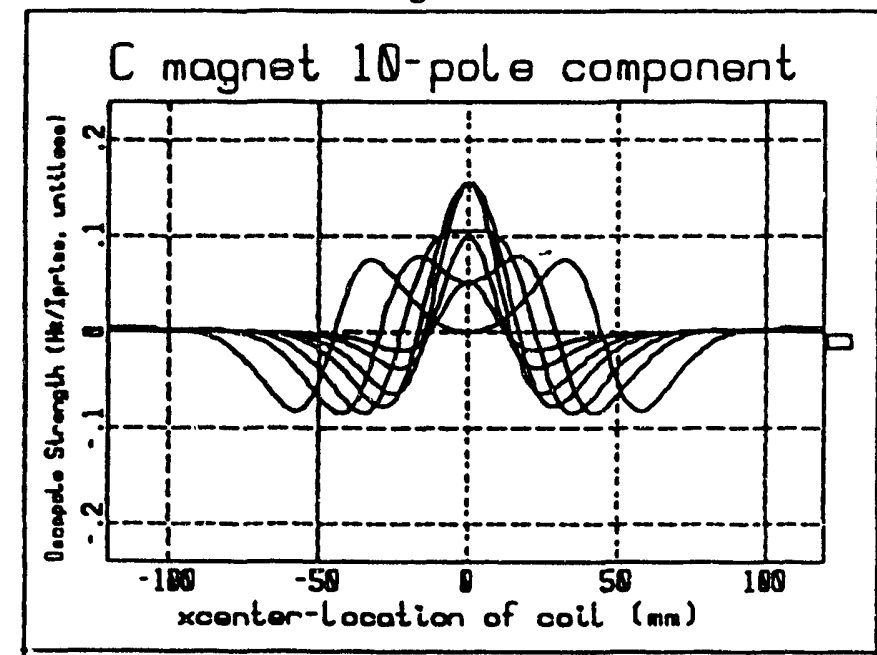

C magnet 12-pole component

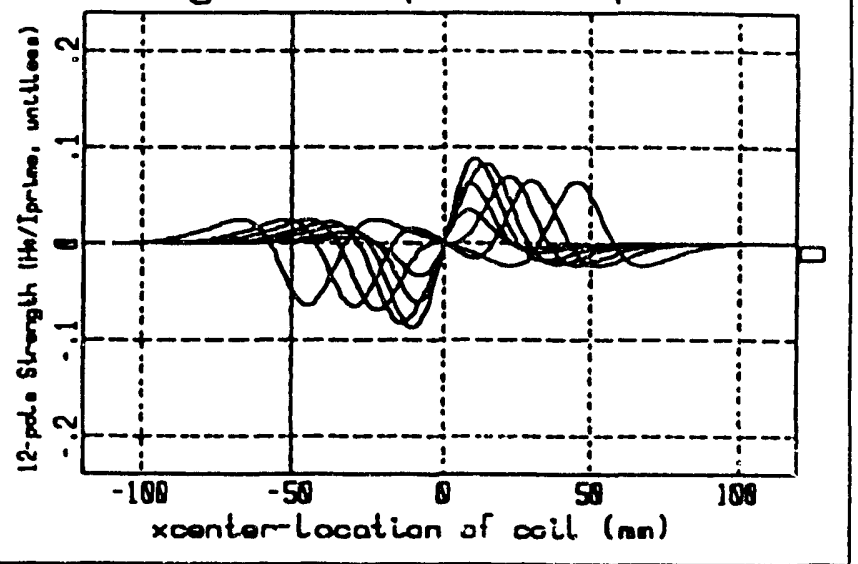

C magnet 14-pole component

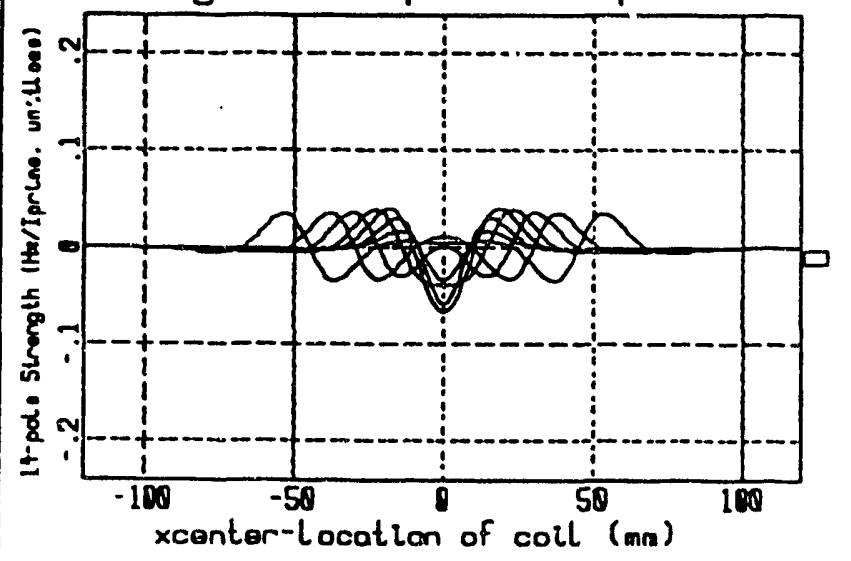

for analytical calculation with $r=30 \mathrm{~mm}, \mathrm{~h}=57.15 \mathrm{~mm}, y_{\text {coils }}=29.01,37.05,45.09,53.13 \mathrm{~mm}$ curves are for $w_{c o 1} 1 s=90,60,45,30,20,10,5 \mathrm{~mm}$ (widest co1l has peaks furthest off-axis) 
Figure 15. Skew harmonics strength dependence on coil position

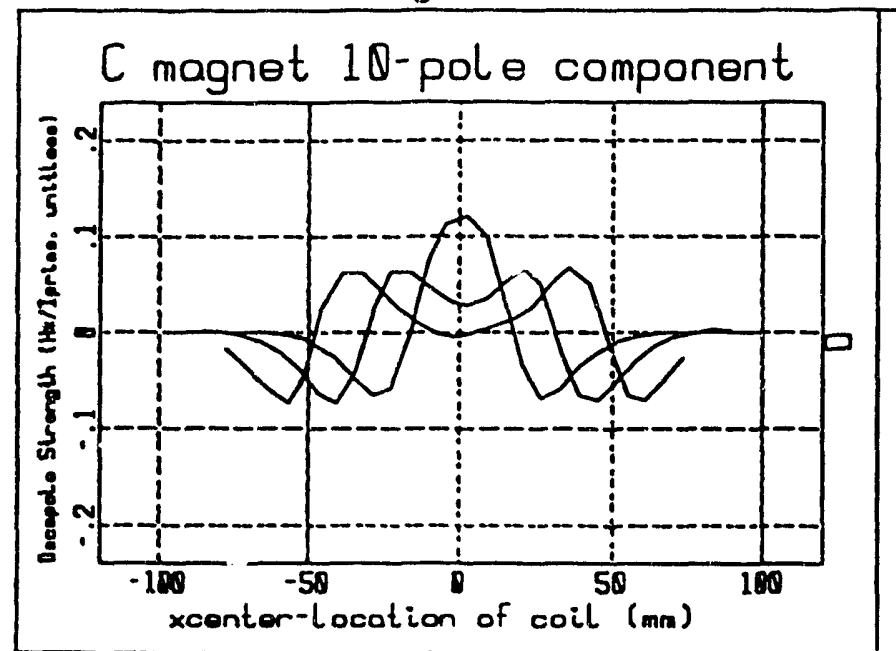

C magnet 12-pole component

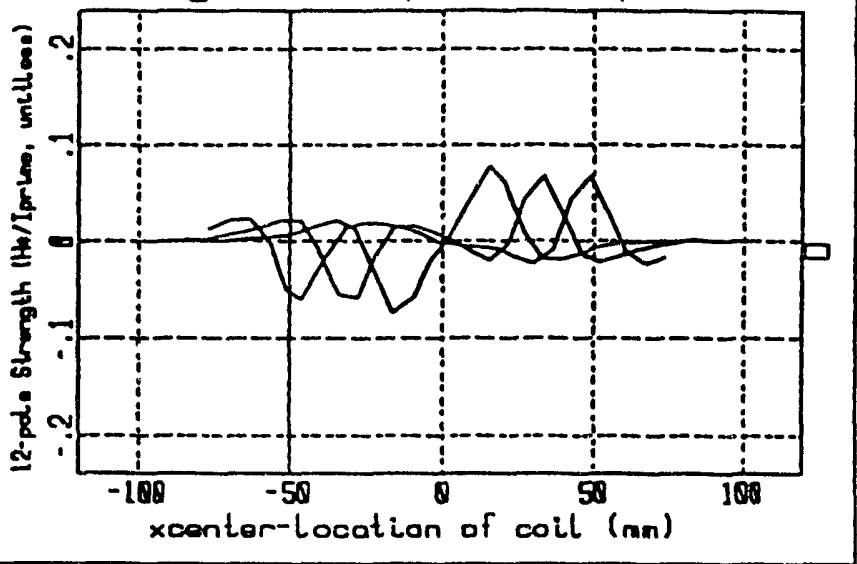

C magnet 14-pole component

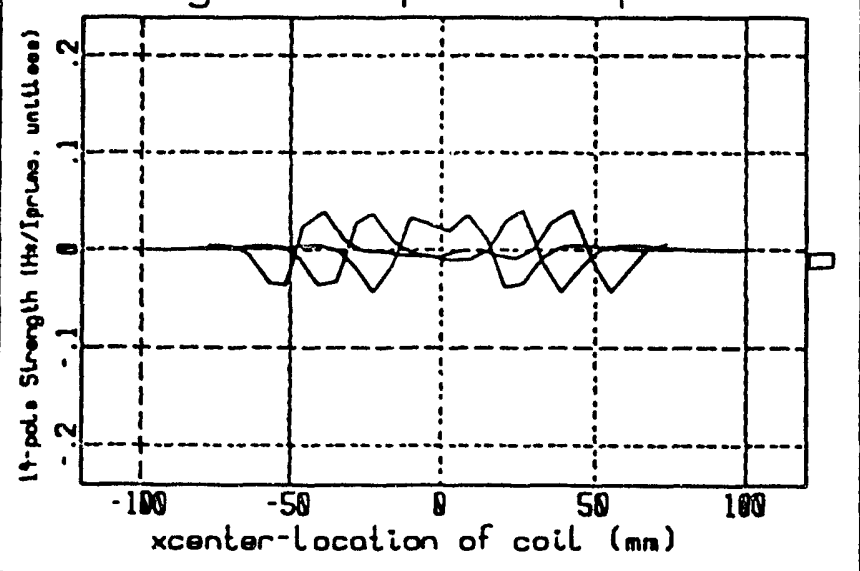

for experimental data with $r=30 \mathrm{~mm}, h=57.15 \mathrm{~mm}, y_{\text {coils }}=29.01,37.05,45.09,53.13 \mathrm{~mm}$ curves are for $w_{\text {colls }}=94.20,62.80,31.40 \mathrm{~mm}(12,8,4$ turns), (widest coil has peaks furthest off-axis) 
Referring to Figure 15, what could have been expected for the 10-, 12-, and 14-pole strengths for the configuration \#7 position? My guess: $\sim-2 \%, \sim 0 \%$, and $\sim-1 \%$. See Figure 8 for the actual experimental results.

Quiz: If it were desired in addition to null the 10-pole component, what would be an expedient manner to proceed?

\section{Acknowledgements}

I am happy to acknowledge the help of Klaus Halbach who provided the analytical foundation for this work. Nord Andresen directed all the experimental work reported herein, Don Lester, Frank Zucca, and Tom Harten worked on the experimental setup, and Dave Van Dyke and Don Nelson made the experimental measurements and handled the data acquisition.

\section{Literature Cited}

1. Jackson, A., Magnetic Field Tolerances in the ALS Storage Ring, ESG Tech Note103, LSAP-56, Jan. 6, 1989.

2. Milburn, J., personal communication.

3. Halbach, K., personal communication.

4. Halbach, K., personal communication. 
Appendix A: Variation of Matrix Elements 


\section{Appendix A: Variation of Matrix Elements}

One must insure that variations in the elements of $[M]$ over the possible excursions of the packettes from their base positions will not cause the the matrix to become singular, and thus the problem as given, intractable. How far can an element $M_{i, j}$ of the matrix $[M]$ be perturbed before the matrix becomes singular ${ }^{(4)}$ ?

Define $\vec{S} \equiv \vec{H}^{*}-\vec{H}_{b a s e}^{*}$, then from Eqns. (14) and (16) we have:

$$
\vec{S}=[M] \Delta \vec{x} \quad \text { and } \quad \Delta \vec{x}=\left[M^{-1}\right] \vec{S}
$$

Let $D$ be the determinant of matrix $[M]$. If element $M_{i, j}$ is perturbed by an amount $\Delta M_{i, j}$, the determinant of perturbed matrix $[M+\Delta M]$ is $D+\Delta D=D+\Delta M_{i, j} C_{i, j}$. where $C_{i, j}$ is the cofactor of element $\underline{M}_{i, j}$. This perturbed matrix will become singular if:

$$
D+\Delta M_{i, j} C_{i, j}=0
$$

The value of $\Delta M_{i, j}$ for the matrix to become singular is thus:

$$
\Delta M_{i, j}=\frac{-D}{C_{i, j}}=\frac{-1}{C_{i, j} / D}=\frac{-1}{M_{j, i}^{-1}}
$$

where use of the relation $M_{j, i}^{-1}=\frac{C_{i}}{D}$ has been made, wic.-- the $M_{j, i}^{-1}$ are elements of the inverse matrix $\left[M^{-1}\right]$.

Application of Eqn. (23) to the matrix $[M]$ given by Eqn. (17) for the final design prototype coil packette configuration shows that if altered one at a time, elements $M_{i, j}$ would have to take on the values given below for the matrix to become singular:

\begin{tabular}{|l|rrr|}
\hline & $i=1$ & $i=2$ & $i=3$ \\
\hline$j=1$ & -0.225 & -0.427 & -0.000 \\
$j=2$ & -0.009 & 0.019 & 0.019 \\
$j=3$ & 0.009 & 0.008 & 0.205 \\
\hline
\end{tabular}

Since one sees (refer to Figure 4) that expected perturbations of coil packette positions necessary to drive to zero the unwanted harmonic components will not cause any of the matrix elements to change anywhere near the amount that would cause the matrix $[M]$ to become singular, the design is robust.

Likewise, application of Eqn. (23) to the matrix $[M]$ given by Eqn. (18) for the final production coil packette configuration reveals another robust design; if altered one at a time, the elements $M_{i, j}$ would have to take on the following values for the matrix to become singular:

\begin{tabular}{|r|rrr|}
\hline & $i=1$ & $i=2$ & $i=3$ \\
\hline$j=1$ & -0.063 & 0.266 & -0.000 \\
$j=2$ & -0.025 & 0.004 & 0.025 \\
$j=3$ & 0.004 & 0.008 & 0.013 \\
\hline
\end{tabular}




\section{Appendix B: Scaling: Experimental versus Analytical Magnitudes}




\section{Appendix B: Scaling: Experimental versus Analytical Magnitudes}

In the analytical work, harmonic strength results were presented in the Figures $3,4,6,7$, and 9 in dimensionless form:

$$
\frac{H^{*}(z=0)}{I^{\prime}} \quad\left(\frac{\text { Amps } / m m}{A m p s ! m m}=\text { unitless }\right)
$$

(In this appendix $z$ is the coordinate in the axial direction, rather than the complex variable $z=x+i y$ used in the analytical sections). Recall that in the analytical work all functions, including $H^{*}$ and $I^{\prime}$ are two-dimensional.

In the experimental work, the integrated (over the axial dimension) field, $\left\langle B^{*} L_{\text {eff }}\right\rangle$ (T-m) and coil current $I$ (Amps) are measured. The expression

$$
\frac{<B^{*} L_{e f f}>10^{6}}{n_{\text {coils }} I} \quad\left(\frac{A m p}{A m p}\right)
$$

is also unitless. For comparison with analytical field stregth calculations however, the numerator and denominator of this expression must be divided by the effective coil length in the axial direction $L_{e f f}$ and the [x-direction] width of the coils $w_{\text {coils }}$ (171.5 mm for the production coils), respectively:

$$
\frac{<B^{*} L_{e f f}>/ L_{e f f}}{n_{\text {coils }} I / w_{\text {coils }}} \cdot \frac{10^{6}}{.4 \pi} \quad\left(\frac{A m p / m m}{A m p / m m}\right)
$$

where $L_{e f f}$ is defined:

$$
L_{e f f}=\frac{\left.\int_{-\infty}^{\infty} H^{*}(0,0, z)\right|_{l=l_{\text {coil }}} d z}{\left.H^{*}(0,0,0)\right|_{l=\infty}}
$$

where $l_{\text {coil }}$ is the effective physical length in the axial direction of the coils. In the denominator of Eqn. (27) the field at the origin is evaluated for $l=\infty$ so as to make it two-dimensional and thus make the analytical and experimental expressions given by Eqns. (24) and (26), respectively, directly comparable. Note that the [analytically calculable, in free space] numerator and denominator of the expression for $L_{\text {eff }}$ given by Eqn. (27) correspond to $<B^{*} L_{e f s}>$ of Eqn. (26) and $H^{*}(z=0)$ of Eqn. (24), respectively. It remains then, to calculate the value of $L_{\text {eff }}$.

Figure 16 shows the geometry under consideration. The beam axis is in the $z$ direction at $(x, y)=(0,0)$. Coils of width $w_{c o i l s}=2 b$ and length $l_{\text {coil }}$ are situated a distance $\pm a$ from the beam axis. The various dimensions in Figure 16 are related as follows: $d l \sin \theta=r d \theta, \frac{a}{\cos \gamma}=r \sin \theta, \frac{d I}{d x} \equiv I^{\prime}=\frac{I}{3 b}$, and $d x \cos \gamma=\frac{a}{\cos \gamma} d \gamma$. Combining the last two relations yields $\frac{d I}{d \gamma}=\frac{I a}{2 b} \sec ^{2} \gamma$. 


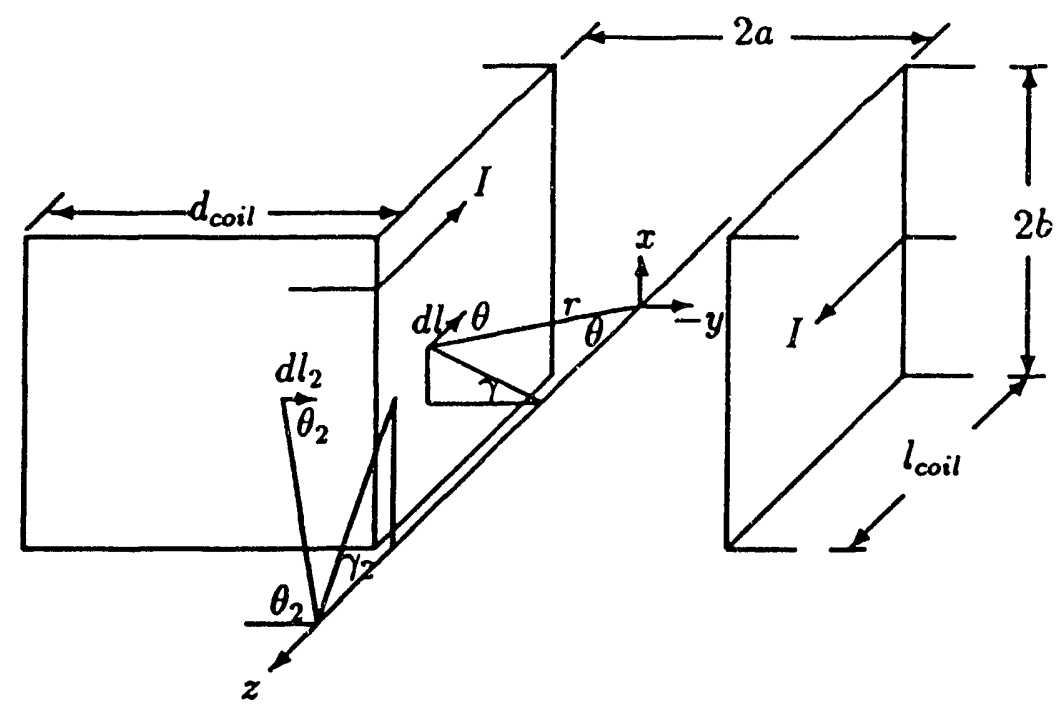

Figure 16. Three-dimensional experimental coil geometry

For a filament segment of length $d l$ along one of the two current sheet faces parallel to the beam axis, the magnetic field at the origin in free-space is given by $\delta H=\frac{\delta I}{4 \pi} \frac{d l \sin \theta}{r^{2}}$. Thus, making use of the eight-fold symmetry, the total magnetic field at the origin is given by

$$
\begin{gathered}
H_{x}(0,0,0)=\frac{8}{4 \pi} \int_{0}^{\arctan \frac{b}{a}} \frac{d I}{d \gamma} \cos \gamma \int_{\arctan \frac{a / \cos \gamma}{c_{\cos } / 2}}^{\frac{\pi}{2}} \frac{d l \sin \theta}{r^{2}} d \theta d \gamma= \\
\frac{8}{4 \pi} \int_{0}^{\arctan \frac{b}{a}} \frac{I a}{2 b} \sec ^{2} \gamma \cos \gamma \int_{\arctan \frac{\pi}{2}}^{\frac{\pi}{l_{\cos i} / 2}} \frac{\cos \gamma \sin \theta}{h} d \theta d \gamma
\end{gathered}
$$

Simplifying gives

$$
\frac{H_{x}(0,0,0)}{I^{\prime}}=\frac{H_{x}(0,0,0)}{I / 2 b}=\frac{2}{\pi} \int_{0}^{\arctan \frac{b}{a}} \frac{\frac{l_{\text {coil }}}{2}}{\sqrt{\left(\frac{\left.I_{\text {cail }}\right)^{2}+\left(\frac{a}{2}\right)^{2}}{\cos \gamma}\right.}} d \gamma
$$

Eqn. (29) gives the value for the denominator of Eqn. (27). More generally, the integrated dipole at location $z$ along the beam axis is similarly given by

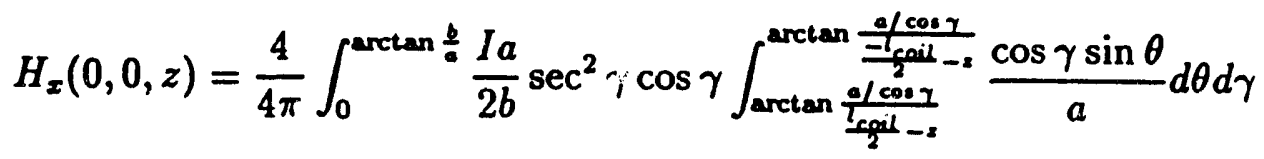

Carrying out the integration over $\theta$ and simplifying gives 


$$
\frac{H_{x}(0,0, z)}{I^{\prime}}=\frac{1}{\pi} \int_{0}^{\arctan \frac{b}{a}}\left[\frac{\frac{l_{\text {coil }}}{2}-z}{\sqrt{\left(\frac{l_{\text {cail }}}{2}-z\right)^{2}+\left(\frac{a}{\cos -)_{i}}\right)^{2}}}-\frac{\frac{-l_{\text {coil }}}{2}-z}{\sqrt{\left(\frac{-l_{\text {cail }}}{2}-z\right)^{2}+\left(\frac{a}{\cos \gamma}\right)^{2}}}\right] d \gamma
$$

Integrating Eqn. (31) over $z$ from $-\infty$ to $\infty$ yields [part of] the value for the numerator of Eqn. (27).

The four current sheet faces perpendicular to the beam axis also contribute significantly to the integral $\int_{-\infty}^{\infty} \frac{H_{x}(0,0, z)}{I^{\prime}} d z$, though not to the on-axis field at locations $|z|<\frac{l_{\text {ceil }}}{2}$, where the iron pole shorts out the flux headed in that direction. U ing quantities $\mathrm{dl}_{2}, r_{2}$, $\theta_{2}, \gamma_{2}$ and $z-\frac{l_{\text {coil }}}{2}$ analogous to $d l, r, \theta, \gamma$ and $a$ above, and making use of eight fold symmetry, we have for the contribution of these current sheet faces to the integral of the magnetic field along the $z$-axis for $|z|>\frac{l_{\text {coil }}}{2}$ :

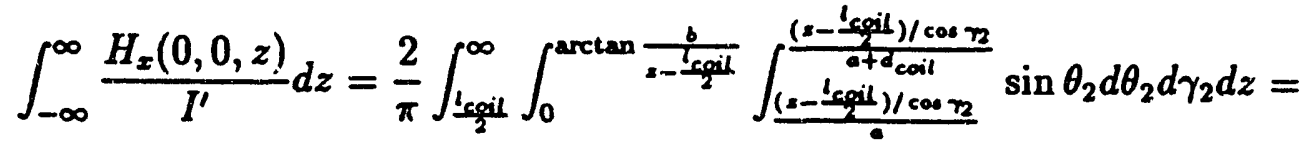

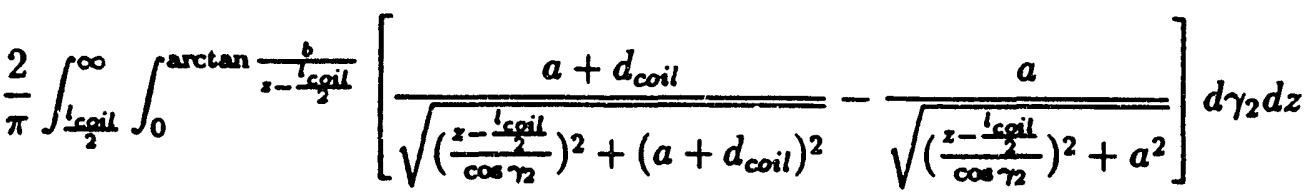

Eqn. (32) constitutes the remainder of the value for the numerator of Eqn. (27). The values and integrals of $H$ along the beam axis and $L_{\text {eff }}$ for various prototype and production coil configurations are shown in Figure 16.

For the ALS storage ring corrector magnets with $w_{\text {coils }} \equiv 2 b=94.2 \mathrm{~mm}, a \equiv$ $y_{\text {coilo }} \simeq 29,37,45,53 \mathrm{~mm}$, with corresponding $l_{\text {coil }} \simeq 193,178,163,148 \mathrm{~mm}, d_{\text {coil }} \simeq$ $270,255,240,225 \mathrm{~mm}$, and $2 h \simeq 114 \mathrm{~mm}$ are the parameters for the first (i.e., $w_{\text {coils }}=$ $94.2 \mathrm{~mm}$ ) of the three $Q, S, O$ sets of experimental curves in Figure 5. For this parameter set, the contribution from the current sheet faces parallel to the beam axis to the numerator and denominator of Eqn. (27) are $\left.\int_{-\infty}^{\infty} \frac{H_{x}(0,0, x)}{I}\right|_{l=l_{\text {coil }}} d z=506$ and $\left.\frac{H_{s}(0,0,0)}{I^{\prime}}\right|_{l=\infty}=2.96$, respectively. The contribution from current sheet faces perpendicular to the beam axis to to the numerator of Eqn. (27) depends on the value of $p$ at which the integration is truncated. $\left.\int_{-p}^{p} \frac{H_{s}(0,0, z)}{p}\right|_{l=l_{\text {coil }}} d z=250$, with $p=400 \mathrm{~mm}$ to simulate the sampling area of the rotating coil of the experimental measurement system. Thus for $w_{\text {coils }}=94.2 \mathrm{~mm}$ we have from Eqn. (27), $L_{\text {eff }}=255 \mathrm{~mm}$.

For the parimeter set of the third (i.e., $w_{\text {coils }}=31.4 \mathrm{~mm}$ ) of the three $Q, S, O$ sets of experimental curves in Figure 5, the values for parallel faces are: $\int_{-\infty}^{\infty} \frac{H_{x}(0,0, z)}{I^{\prime}} d z=294$ and $\frac{H_{z}(0,0,0)}{I^{\prime}}=1.70$, and for perpendicular faces: $\left.\int_{-p}^{p} \frac{H_{z}(0,0, z)}{I^{\prime}}\right|_{l=l_{\text {coil }}} d z=99$, with with $p=400 \mathrm{~mm}$ to simulate the sampling area of the rotating coil of the experimental measurement system. Thus for $w_{\text {coils }}=31.4 \mathrm{~mm}$ we have from Eqn. (27), $L_{\text {efs }}=231$ $\mathrm{mm}$.

These free-space results will differ somewhat from actual experiment-analytical comparison due to differential effects of the iron in the two geometries (3-D actual iron.pole 
Figure 16. Values and integrals of $H$ along the beam axis and resulting $L_{\text {eff }}$

\begin{tabular}{|c|c|c|c|c|c|c|c|c|c|c|}
\hline & $a / l_{\text {coil }} / d_{\text {coil }}$ & \multicolumn{4}{|c|}{$\int_{-p}^{p} \frac{H_{x}(0,0, z)}{I^{\prime}} d z$} & \multicolumn{2}{|c|}{$\frac{H_{x}(0,0,0)}{I^{\prime}}$} & \multicolumn{3}{|c|}{$L_{\text {esf }}$} \\
\hline & $\mathrm{mm} / \mathrm{mm} / \mathrm{mm}$ & faces & & faces & & $l=l_{\text {coil }}$ & $l=\infty$ & & $\mathrm{mm}$ & \\
\hline$p(\mathrm{~mm}):$ & & $\infty$ & $\sim \infty$ & 400 & 222 & & & $\sim \infty$ & 400 & 222 \\
\hline $\begin{array}{l}\text { prototype coils, } \\
2 b=94.2 \mathrm{~mm}\end{array}$ & $\begin{array}{l}29 / 193 / 270 \\
37 / 178 / 255 \\
45 / 163 / 240 \\
53 / 147 / 225\end{array}$ & $\begin{array}{r}156 \\
135 \\
116 \\
99\end{array}$ & $\begin{array}{l}96 \\
93 \\
85 \\
79\end{array}$ & $\begin{array}{l}72 \\
65 \\
59 \\
54\end{array}$ & $\begin{array}{l}40 \\
34 \\
33 \\
30\end{array}$ & $\begin{array}{l}.736 \\
.659 \\
.580 \\
.501\end{array}$ & $\begin{array}{l}.810 \\
.762 \\
.716 \\
.674\end{array}$ & & & \\
\hline$\Sigma:$ & & 506 & 352 & 250 & 137 & & 2.960 & 290 & 255 & 217 \\
\hline $\begin{array}{l}\text { prototype coils, } \\
2 b=31.4 \mathrm{~mm}\end{array}$ & $\begin{array}{l}29 / 193 / 270 \\
37 / 178 / 255 \\
45 / 163 / 240 \\
53 / 147 / 225\end{array}$ & $\begin{array}{r}101 \\
80 \\
63 \\
50\end{array}$ & $\begin{array}{l}38 \\
35 \\
32 \\
29\end{array}$ & $\begin{array}{l}30 \\
26 \\
23 \\
20\end{array}$ & $\begin{array}{l}18 \\
14 \\
12 \\
10\end{array}$ & $\begin{array}{l}.497 \\
.408 \\
.334 \\
.272\end{array}$ & $\begin{array}{l}.525 \\
.448 \\
.388 \\
.340\end{array}$ & & & \\
\hline$\sum:$ & & 294 & 133 & 99 & 54 & & 1.700 & 251 & 231 & 205 \\
\hline $\begin{array}{l}\text { production coils, } \\
2 b=170 \mathrm{~mm}\end{array}$ & $\begin{array}{l}34 / 189 / 266 \\
40 / 175252 \\
47 / 161238 \\
54 / 147 / 224\end{array}$ & $\begin{array}{l}165 \\
149 \\
132 \\
117 \\
\end{array}$ & $\begin{array}{l}142 \\
140 \\
130 \\
123 \\
\end{array}$ & $\begin{array}{l}97 \\
91 \\
84 \\
78\end{array}$ & $\begin{array}{l}39 \\
35 \\
36 \\
34\end{array}$ & $\begin{array}{l}.749 \\
.692 \\
.626 \\
.558\end{array}$ & $\begin{array}{l}.874 \\
.853 \\
.828 \\
.804\end{array}$ & & & \\
\hline$\Sigma:$ & & 562 & 534 & 350 & 145 & & 3.360 & 326 & 271 & 210 \\
\hline
\end{tabular}


versus 2-D infinite iron pole) and due to the approximate nature of the geometry of Figure 16. The actual values of $L_{\text {eff }}$ required to make the analytical (cf. Eqn (13). Figure 3 )) and prototype experimental values (not shown in Figure 5) of the dipole component of $H(0,0,0)$ agree for the two cases above are 262 and 240 , respectively. Thus it is seen that the predictions above, $L_{\text {eff }}=255$ and 231 , for the two cases, respectively, with a \pm 400 $\mathrm{mm}$ cutoff in $z$ on the integrated fields to match the rotating coil length, are extremely close to the experimentally obtained values. $L_{\text {eff }}=250$ was used in the normalization of all the experimental data shown in Figure 5.

What are the implications of this lengthy three-dimensional $L_{\text {eff }}$ analysis? A sizeable portion (25-50\%) of the integrated dipole is shown to be due to the current sheet faces perpendicular to the beam axis. Higher order harmonics are also affected, though not necessarily proportionately. This accounts for minor magnitude scaling differences between the analytical and experimental harmonic magnitudes (cf. Figures 4 and 5). It also illustrates how placement of iron quadrupole cores 6" from the $C$-magnet (i.e. at $z=$ $\pm 222 \mathrm{~mm}$ ) affects the harmonics as well as the integrated dipole. For the production coil designs of Figure 8, placement of the adjacent quadrupoles cores at $z= \pm 222 \mathrm{~mm}$ can be expected (see Figure 16) to decrease the measured integrated dipole to $77 \%$ $\left(=\frac{562+145}{562+350}\right)$ of its original value without the cores present, assuming that the cores shunt all the flux at locations $z>222 \mathrm{~mm}$ through the iron, rather than across the beam axis. Experimentally, the measured integrated dipole drops to $76 \%$ of its original value when the adjacent quadrupole cores are in position. The analytical predictions are remarkably accurate. From Figure 16, it is also seen that for the isolated $C$-magnet, only $\simeq 83 \%$ $\left(=\frac{562+350}{562+534}\right)$ of the total integrated field is being measured by the $\simeq 80 \mathrm{~mm}$ long rotating measurement coil. 


\section{Appendix C: Computer Codes}

Files cmagplot.for and cmagmate. for can be found in LBL VAX directory _DSA123:[ROSS.CMAG_ALS]. 

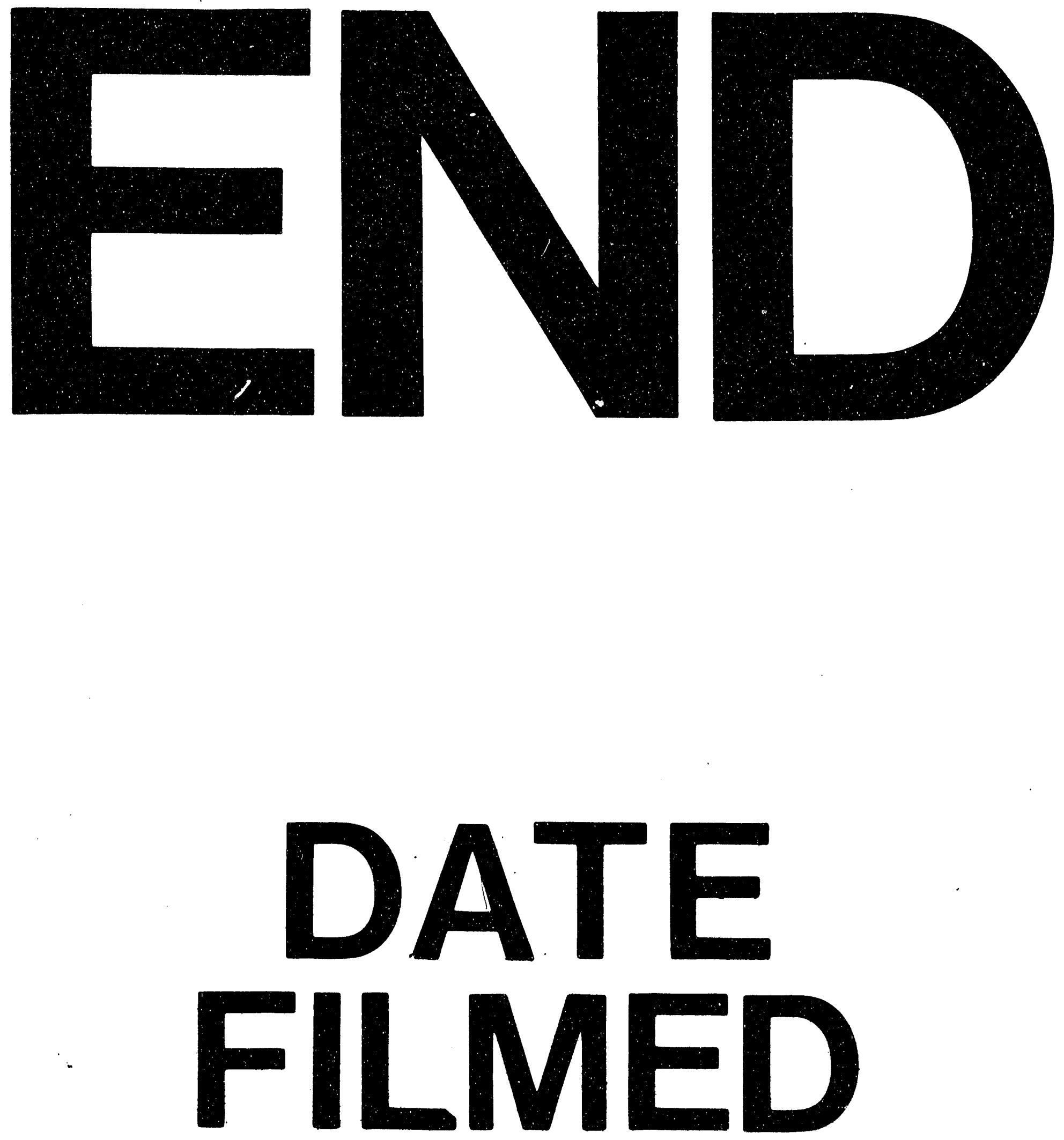

II

1

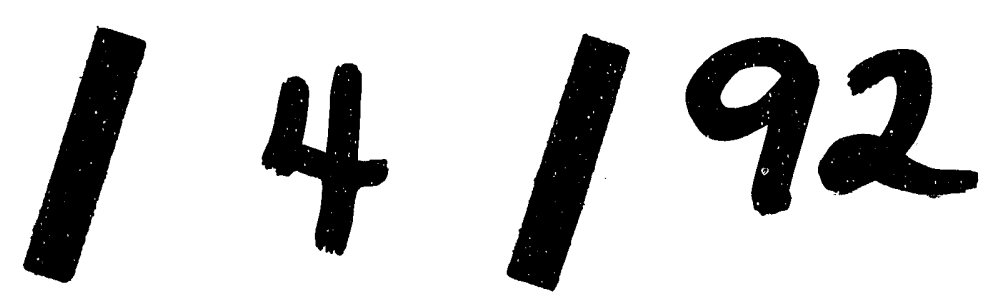


